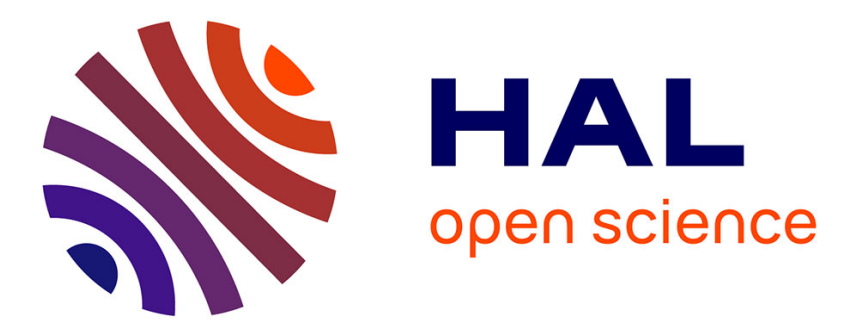

\title{
Assessing temperature effects on multipole contributions and angular dependence in core-level spectroscopies
}

S. Delhommaye, G. Radtke, Ch. Brouder, S. P Collins, S. Huotari, Ch. Sahle, M. Lazzeri, L. Paulatto, D. Cabaret

\section{- To cite this version:}

S. Delhommaye, G. Radtke, Ch. Brouder, S. P Collins, S. Huotari, et al.. Assessing temperature effects on multipole contributions and angular dependence in core-level spectroscopies. Physical Review B, 2021, 104 (2), 10.1103/PhysRevB.104.024302 . hal-03365381

\section{HAL Id: hal-03365381 \\ https://hal.science/hal-03365381}

Submitted on 5 Oct 2021

HAL is a multi-disciplinary open access archive for the deposit and dissemination of scientific research documents, whether they are published or not. The documents may come from teaching and research institutions in France or abroad, or from public or private research centers.
L'archive ouverte pluridisciplinaire HAL, est destinée au dépôt et à la diffusion de documents scientifiques de niveau recherche, publiés ou non, émanant des établissements d'enseignement et de recherche français ou étrangers, des laboratoires publics ou privés. 


\title{
Assessing temperature effects on multipole contributions and angular dependence in core-level spectroscopies
}

\author{
S. Delhommaye, ${ }^{1}$ G. Radtke, ${ }^{1, *}$ Ch. Brouder, ${ }^{1}$ S.P. Collins,${ }^{2}$ \\ S. Huotari, ${ }^{3}$ Ch. Sahle, ${ }^{4}$ M. Lazzeri, ${ }^{1}$ L. Paulatto, ${ }^{1}$ and D. Cabaret $^{1}$ \\ ${ }^{1}$ Sorbonne Université, Muséum National d'Histoire Naturelle, \\ UMR CNRS 7590, IRD, Institut de Minéralogie, \\ de Physique des Matériaux et de Cosmochimie, IMPMC, 75005 Paris, France \\ ${ }^{2}$ Diamond Light Source Ltd, Diamond House, Harwell Science and Innovation Campus, Didcot OX11 ODE, United Kingdom \\ ${ }^{3}$ Department of Physics, University of Helsinki, FI-00014 Helsinki, Finland \\ ${ }^{4}$ European Synchrotron Radiation Facility, 38043 Grenoble, France
}

\begin{abstract}
This study aims at assessing the thermal nuclei motion effects on the multipole transition channels involved in two core-level spectroscopies, X-ray Absorption Spectroscopy (XAS) and X-ray Raman Scattering (XRS). Temperature effects on the $1 s \rightarrow s$ monopole, $1 s \rightarrow p$ dipole and $1 s \rightarrow d$ quadrupole transitions are investigated using two reference systems for which we present original experimental data: $\alpha-\mathrm{Al}_{2} \mathrm{O}_{3}$ at the $\mathrm{Al} K$ edge probed by XRS at room temperature and rutile $\mathrm{TiO}_{2}$ at the Ti $K$ pre-edge probed by XAS at temperatures ranging from $6 \mathrm{~K}$ to $700 \mathrm{~K}$. Through the latter, this work enlightens the part of the pre-edge peak enhancement due to temperature in the $K$ pre-edge region of $3 d$ transition metal, which is known to be routinely used to determine the concentration, valence or symmetry of the probed element in a given sample. Nuclear thermal fluctuations are taken into account using a method based on density functional theory that consists in averaging spectra over atomic configurations, generated within the harmonic approximation and obeying quantum statistics at finite temperature. Since only a finite number of such configurations are used, the numerically averaged spectra generally lose the symmetry of the equilibrium crystal positions. In this paper, we demonstrate that the physical average has to be symmetric and propose a method to restore the physical angular dependence of the spectra. The approach is successfully applied to investigate the angular dependent XAS spectra in rutile as a function of temperature. The two systems under study allow to draw general conclusions regarding the effect of nuclear quantum fluctuations on the different transition channels available to both core-level spectroscopies.
\end{abstract}

\section{INTRODUCTION}

Temperature-dependent measurements are widely employed in X-ray Absorption Spectroscopy (XAS) to monitor the evolution of the local atomic and electronic structure of materials undergoing phase transitions, such as superconductors, ${ }^{1-4}$ colossally magnetoresistive manganites, ${ }^{5-7}$ ferroelectrics ${ }^{8-10}$ or thermochromics. ${ }^{11,12}$ While XAS still appears as a technique of reference, Xray Raman Scattering spectroscopy (XRS) is becoming increasingly popular in materials and earth sciences for conducting in-situ experiments. This is mostly due to its bulk sensitivity, its ability to probe low-energy edges and thus, light elements, and to its high flexibility in terms of sample environments. ${ }^{13,14}$ Indeed, this non-resonant spectroscopy is based on the inelastic scattering of hard $\mathrm{X}$-rays and therefore permits the use of highly absorbing experimental cells for pressure ${ }^{15-18}$ and temperature ${ }^{19-21}$ dependent measurements, which are often hindered by the use of soft X-rays in XAS.

Temperature appears therefore as a key parameter in many studies based on core-level spectroscopies. Understanding how atomic thermal fluctuations can influence the shape of XAS or XRS spectra is, consequently, of fundamental importance to interpret these data accurately. To this end, strategies have been developped to account theoretically for atomic vibrations when modeling the spectra. Methods that do not require the explicit calculation of phonon modes have been implemented first, either based on displacing the core-state orbital only while treating final states within the crude BornOppenheimer approximation, ${ }^{22,23}$ displacing the entire absorbing atom around its equilibrium position ${ }^{24,25}$ or convoluting the spectrum with an approximate phonon spectral function. ${ }^{24,26}$ However, heavier numerical approaches based on averaging spectra calculated for series of vibrationally distorted structures generated either from snapshots of molecular dynamics simulations ${ }^{20,27,28}$ (where the nuclear dynamics is always classic) or from the phonon quantum dynamics ${ }^{29-31}$ were later preferred and proved to be necessary for capturing the subtle effects of temperature on the spectral shape.

In this context, it would be interesting to evaluate the impact of atomic quantum thermal fluctuations on the different transition channels observed at $K$ edges in X-ray Absorption and X-ray Raman spectroscopies. $K$ pre-edge XAS spectra of $3 d$ transition metal ions are indeed built from a sum of electric dipole and quadrupole transitions. ${ }^{32}$ Whereas the former give access to the absorber unoccupied $p$ states and thus, to the $3 d$ states only indirectly through off-site and, possibly, on-site hybridization, the latter provide a direct channel for probing the absorber $3 d$ final states. ${ }^{33}$ Along the same line, under certain scattering conditions where large momenta are transferred to the specimen, electronic transitions observed in $K$ pre-edge XRS spectra of light elements 
can strongly deviate from the standard electric dipole channel $^{34}$ to include monopole transitions and therefore probe selectively final states with $s$ or $p$ character. ${ }^{35,36}$ Although the impact of nuclear quantum fluctuations on electric dipole transitions has been investigated in a number of studies, ${ }^{23,24,30,31}$ little is known about their influence on these secondary transition channels. Here, we present an in-depth experimental and theoretical investigation of the Ti $K$ pre-edge temperature dependence in rutile $\mathrm{TiO}_{2}$. In particular, we extend the numerical approach developed in previous works to account for lattice quantum fluctuations ${ }^{30,31}$ to the calculation of a weak dichroic signal on the basis of group-theoretical arguments. We also consider the case of the $\mathrm{Al} K$ edge in corundum $\alpha-\mathrm{Al}_{2} \mathrm{O}_{3}$ measured in XRS at $300 \mathrm{~K}$ and for a norm of the scattering vector close to $10 \AA^{-1}$ to assess the effect of temperature on both electric monopole and dipole transitions with this spectroscopy.

The paper is organized as follows. Section II briefly introduces the theory of X-ray absorption and X-ray Raman spectroscopies, provides a short overview of the method employed to account for lattice quantum dynamics, which is based on density functional theory (DFT), and presents the experimental and computational details related to this work. A detailed presentation of the group theoretical approach devised to extract the dichroic signal from a set of calculations performed when accounting for atomic displacements is given in Appendix B. In section III, we present a systematic comparison between measured and calculated spectra obtained at finite temperatures for the Ti $K$ edge in rutile $\mathrm{TiO}_{2}$ in XAS and the $\mathrm{Al} K$ edge in $\alpha-\mathrm{Al}_{2} \mathrm{O}_{3}$ in XRS. In Section IV finally, we present our conclusions on the influence of temperature on multipole transitions and orientation dependence in core-level spectroscopies.

\section{METHODS}

\section{A. General framework}

In core-electron excitation spectroscopies such as XAS and XRS, the spectrum modelling involves the calculation of transition matrix elements, $M_{i \rightarrow f}$, between an initial state $|i\rangle$ and a final state $|f\rangle$ through a transition operator, $\mathcal{O}$, which represents the photon-electron interaction

$$
M_{i \rightarrow f}=\langle f|\mathcal{O}| i\rangle .
$$

In a single-particle DFT-based approach, the $|i\rangle$ initial state, of energy $E_{i}$, is an atomic core-level state (1s orbital for a $K$ edge), the $|f\rangle$ final state, of energy $E_{f}$, is an unoccupied single-particle state calculated for a supercell which includes the core-hole located on the absorbing atom, and the specific form of the operator $\mathcal{O}$ depends on the spectroscopy.

In the case of XAS treated in the electric quadrupole approximation, which is required in the present study,
$\mathcal{O}^{\mathrm{XAS}}$ is the sum of electric-dipole (E1) and electricquadrupole (E2) terms that read, respectively

$$
\begin{aligned}
\mathcal{O}_{\text {dip }}^{\text {XAS }} & =\hat{\varepsilon} \cdot \boldsymbol{r}, \\
\mathcal{O}_{\text {quad }}^{\text {XAS }} & =\frac{\mathrm{i}}{2} \hat{\varepsilon} \cdot \boldsymbol{r} \boldsymbol{k} \cdot \boldsymbol{r},
\end{aligned}
$$

where $\mathrm{i}$ is the imaginary unit, and $\hat{\boldsymbol{\varepsilon}}$ and $\boldsymbol{k}$ are the polarization unit vector and the wave vector of the incident $\mathrm{X}$-ray beam, respectively. For non-magnetic materials and a linear polarization of the x-ray beam, the XAS cross section is the sum of E1 and E2 contributions, ${ }^{37}$ given by

$$
\begin{aligned}
\sigma(\hbar \omega)= & 4 \pi^{2} \alpha_{0} \hbar \omega \\
& \times \sum_{f}\left[|\langle f|\hat{\boldsymbol{\varepsilon}} \cdot \boldsymbol{r}| i\rangle|^{2}+\frac{1}{4}|\langle f|\hat{\boldsymbol{\varepsilon}} \cdot \boldsymbol{r} \boldsymbol{k} \cdot \boldsymbol{r}| i\rangle|^{2}\right] \\
& \times \delta\left(E_{f}-E_{i}-\hbar \omega\right)
\end{aligned}
$$

where $\alpha_{0}$ is the fine structure constant and $\hbar \omega$ is the energy of the incident photon.

In the case of XRS, a non-resonant inelastic photon- $i n$ photon-out scattering experiment, the transition operator is given by

$$
\mathcal{O}^{\mathrm{XRS}}=e^{\mathrm{i} \boldsymbol{q} \cdot \boldsymbol{r}}
$$

where $\boldsymbol{q}=\boldsymbol{k}_{\text {in }}-\boldsymbol{k}_{\text {out }}$ is the scattering vector. The expansion of Eq. 5 in terms of complex spherical harmonics $Y_{l}^{m}$ and spherical Bessel functions $j_{l}$ leads to the XRS monopole and dipole operators defined by

$$
\begin{aligned}
\mathcal{O}_{\text {mono }}^{\mathrm{XRS}} & =j_{0}(q r), \\
\mathcal{O}_{\mathrm{dip}}^{\mathrm{XRS}} & =j_{1}(q r) 4 \mathrm{i} \pi \sum_{\lambda}(-1)^{\lambda} Y_{1}^{-\lambda}(\hat{\boldsymbol{q}}) Y_{1}^{\lambda}(\hat{\boldsymbol{r}}),
\end{aligned}
$$

As shown in Ref. 35, the contribution of the XRS quadrupole term can be safely neglected when investigating $K$ edges of light elements. The monopole XRS operator depends on the norm $q$ of vector $\boldsymbol{q}$, while the dipole operator depends on both its norm and direction $\hat{\boldsymbol{q}}$. When considering powder specimens, cross-term cancel and the XRS dynamic structure factor can be seen as a sum of two distinct transition-channel contributions ${ }^{35}$

$$
\begin{aligned}
S(\boldsymbol{q}, \hbar \omega)= & \sum_{f}\left[\left|\left\langle f\left|\mathcal{O}_{\mathrm{mono}}^{\mathrm{XRS}}\right| i\right\rangle\right|^{2}+\left|\left\langle f\left|\mathcal{O}_{\mathrm{dip}}^{\mathrm{XRS}}\right| i\right\rangle\right|^{2}\right] \\
& \times \delta\left(E_{f}-E_{i}-\hbar \omega\right),
\end{aligned}
$$

where $\hbar \omega=\hbar \omega_{\text {in }}-\hbar \omega_{\text {out }}$ is the energy transfer. Whether in XAS or XRS, the monopole, dipole and quadrupole operators each select a specific electronic transition, i.e. $1 s \rightarrow s$ (forbidden in XAS), $1 s \rightarrow p$ and $1 s \rightarrow d$, respectively.

A method has recently been developed by Nemausat et $a l .{ }^{30,31}$ to account for temperature effects in the calculation of XAS or XRS spectra. Within this method 
the spectra are obtained by averaging on non-equilibrium atomic configurations that obey quantum statistics at finite temperature. The configurations are obtained from the atomic vibrations within the framework of both the Born-Oppenheimer and the (quasi)-harmonic approximations. The method is based on a three-step process: (i) the atomic vibrational modes (phonons) of the supercell used in the calculation of the spectra are determined by using DFT; ${ }^{38}$ (ii) for a given temperature, a set of configurations is then generated by introducing random displacements of the nuclear positions in the supercell following the procedure described in Ref. 31; (iii) the XAS and XRS spectra are finally calculated for each atomic configuration generated in the previous step and averaged to get a spectrum to be compared to experiment. The convergence of the average spectrum is achieved as a function of the number of configurations. The validity of this approach has been demonstrated in the Appendix of Ref. 30, showing in particular that the use of an average is only justified if the nuclear kinetic energy is neglected.

\section{B. Experimental details}

Ti $K$-edge spectra of rutile $\mathrm{TiO}_{2}$ were measured at the I16 beam line of the Diamond Light Source using a synthetic single crystal. Rutile $\mathrm{TiO}_{2}$ crystallizes in the tetragonal system with space group $P 4_{2} / m n m$ (n. 136), the 4 -fold symmetry axis being parallel to the [001] direction. The experimental setup was designed to study the angular and temperature dependence of the pre-edge region of the Ti $K$ edge by using a resonant elastic x-ray scattering geometry, as shown in Fig. 1a. The sample $(10 \times 10 \times 1 \mathrm{~mm}$ square $)$ was mounted on the cold finger of a closed-cycle ARS cryofurnace and oriented by x-ray diffraction. Sample rotations about the [001] axis were carried out while maintaining angles of 10 and 70 degrees between the sample surface, and incident and scattered beams, respectively, in the scattering $\sigma$ polarization configuration (polarization vector perpendicular to the scattering plane). In this setup geometry, polarization vector $\hat{\varepsilon}$ was kept normal to the 4 -fold symmetry axis of the crystal, so that only electric-quadrupole angular dependence was expected. ${ }^{37}$ The angular-dependent spectra were recorded for different temperatures ranging from $6 \mathrm{~K}$ to $698 \mathrm{~K}$, by detecting the total fluorescence signal using a Pilatus $100 \mathrm{~K}$ area detector. Fig. 1b displays XAS spectra in the pre-edge region recorded at $6 \mathrm{~K}$ for a $\pi$ rotation of azimuthal angle $\varphi$. The A1 peak, known to be due to E2 transitions, ${ }^{39}$ does exhibit the expected $\pi / 2$ periodicity. ${ }^{37}$ The maximum and minimum intensities of peak A1 define the two spectra that are considered in the following. Their mean and difference are denoted E2 in-plane mean spectrum and E2 in-plane dichroic signal, respectively. Self-absorption correction were applied using the process described in Ref. 40.

The Al $K$-edge XRS spectrum was recorded at room temperature on a powder sample of $\alpha-\mathrm{Al}_{2} \mathrm{O}_{3}$. The
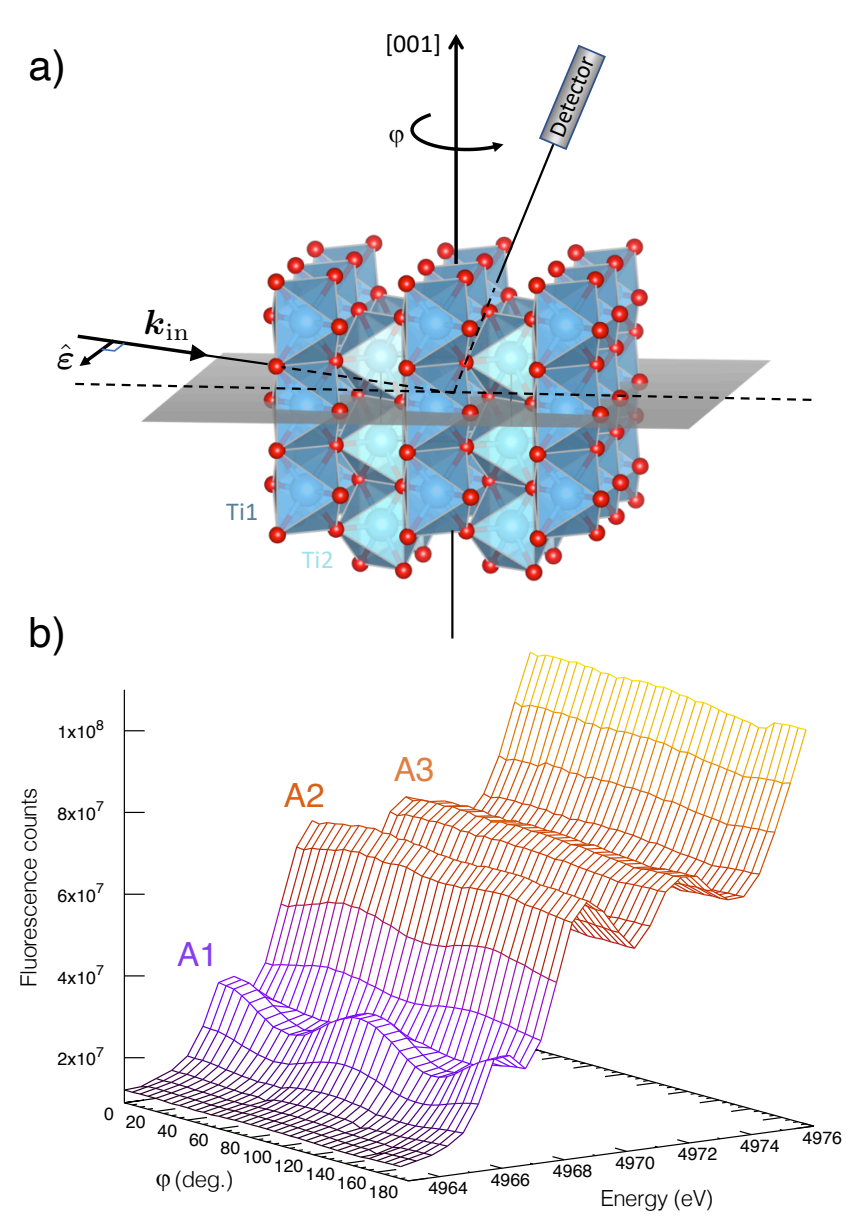

FIG. 1: a) Experimental setup used to record the polarization-dependent x-ray absorption spectra at the Ti $K$ edge of $\mathrm{TiO}_{2}$ rutile, as a function of temperature. As the polarization remains in the plane perpendicular to the crystal [001] direction, in-plane dichroism only arises from electric quadrupole E2 transitions. b) Ti $K$ pre-edge spectra obtained at $6 \mathrm{~K}$ for azimuthal angle $\varphi$ varying from 0 to $\pi$. The minimum and maximum intensity of peak A1 identify the two $\varphi$ values that are considered in this study to define E2 in-plane mean spectrum and E2 in-plane dichroic signal.

experiment was performed at the ID20 beamline of the European Synchrotron Radiation Facility (ESRF) in Grenoble, France, using the large-solid angle XRS spectrometer. ${ }^{41}$ The synchrotron ring was operating at $200 \mathrm{~mA}$ and the beam was monochromated by a combination of $\mathrm{Si}(111)$ double-crystal cryogenically cooled premonochromator and a $\mathrm{Si}(311)$ channel-cut postmonochromator. The total energy resolution was about $0.6 \mathrm{eV}$. A total of 24 analyzer crystals using the $\mathrm{Si}(660)$ reflection were utilized at a mean momentum transfer of $9.5 \AA^{-1} \pm 0.5 \AA^{-1}$, a high value enhancing monopole transitions. 


\section{Computational details}

Self-consistent field (SCF) charge densities, phonons, XAS and XRS spectra were calculated with the Quantum-ESPRESSO suite of codes, ${ }^{42,43}$ which uses planewave basis set, pseudopotentials and periodic boundary conditions. XAS spectra were calculated with the XSpectra module using the formalism described in Refs. 44,45. The XRS spectra were obtained using a modified version of XSpectra, where the XRS dynamic structure factor has been implemented. ${ }^{35}$ Ultrasoft pseudopotentials ${ }^{46}$ from the PSLIBRARY ${ }^{47}$ were generated in the Rappe, Rabe, Kaxiras, and Joannopoulos formalism. ${ }^{48}$ Each pseudopotential contains two projectors per angular momentum channel whereas Ti $3 s$ and $3 p$ semicore states have been explicitely treated in valence. The pseudopotentials of the absorbing atoms ( $\mathrm{Ti}$ and $\mathrm{Al}$ ) were generated by fixing the occupancy of the $1 s$ core-state to one and by adjusting the ionic radii to slightly smaller values. The latter step is necessary to account for the overall atomic orbital contraction associated with this excited electronic configuration.

For $\alpha-\mathrm{Al}_{2} \mathrm{O}_{3}$, the calculations were performed within the generalized gradient approximation using the Perdew, Burke and Ernzerhof (GGA-PBE) parametrization. ${ }^{49}$ The case of rutile $\mathrm{TiO}_{2}$, an incipient ferroelectric material, is more subtle as GGA-PBE predicts the $A_{2 u}$ mode to be unstable at the equilibrium geometry, ${ }^{50,51}$ leading to a ferroelectric distorsion of the crystal which qualitatively disagrees with the known properties of this compound. The use of the local density approximation (LDA) ${ }^{52}$ however, leads to a stable structure characterized by a slightly smaller equilibrium volume of the unit cell and provides a correct description of its lattice dynamics. This justifies our choice of LDA for the present study of rutile $\mathrm{TiO}_{2}$. For both compounds, the energy cutoffs were set to $50 \mathrm{Ry}$ and $500 \mathrm{Ry}$ for the electronic wave function and density, respectively.

For the pre-edge structure analysis, local and partial densities of states (DOS) were calculated using Löwdin projections in supercells with the atoms at their equilibrium positions, on a $4 \times 4 \times 4 k$-point grid with a Gaussian parameter of $0.5 \mathrm{eV}$. These DOS calculations were performed using the LDA-optimized structure for rutile and the experimental structure at room temperature for corundum. ${ }^{53}$

\section{1. $\mathrm{Al} \mathrm{K}$ edge $\mathrm{XRS}$ in $\alpha-\mathrm{Al}_{2} \mathrm{O}_{3}$}

Phonon calculations were first performed using the room temperature experimental unit cell of $\alpha-\mathrm{Al}_{2} \mathrm{O}_{3} \cdot{ }^{53}$ $\mathrm{A}$ set of atomic configurations were then generated in $2 \times 2 \times 2$ (80 atoms) rhombohedral supercells, large enough to minimize spurious interactions between periodically repeated images of the absorber. All spectra were calculated in the full core-hole $(\mathrm{FCH})$ approximation, where the missing electronic charge on the $1 s$ core orbital is compensated by a uniform negative background in the supercell. Monopole and dipole contributions to the dynamic form factor (see Eq. 8) were calculated as continued fractions ${ }^{35,44}$ with a broadening parameter of $0.3 \mathrm{eV}$, on a $4 \times 4 \times 4$ sampling of the supercell Brillouin zone and for a module of the scattering vector of $10 \AA^{-1}$. A converged spectrum was obtained by averaging the contributions arising from 50 atomic configurations, which were realigned with respect to each other through standard $\triangle$ SCF calculations, ${ }^{54}$ and finally energy shifted to match the experimental main edge peak energy position.

\section{Ti $\mathrm{K}$ edge $\mathrm{XAS}$ in rutile $\mathrm{TiO}_{2}$}

The procedure used for $\mathrm{Al} \mathrm{K}$ edge XRS in $\alpha-\mathrm{Al}_{2} \mathrm{O}_{3}$ has been slightly modified in the case of rutile $\mathrm{TiO}_{2}$ due to the systematic occurence of imaginary frequencies when performing phonon calculations on experimental structures. Indeed, as the quasi-harmonic approximation ${ }^{55}$ is not applicable in this particular case, phonon calculations have been performed on a fully relaxed structure. Atomic configurations were then generated in tetragonal $2 \times 2 \times 3$ (72 atoms) supercells where thermal effects were only taken into account in the calculated nuclear displacements through the temperature dependence of the modes normal length (see Eq. 4 of Ref. 31) whereas the expansion of the lattice parameters with increasing temperatures was neglected. As in the case of $\alpha-\mathrm{Al}_{2} \mathrm{O}_{3}$, spectra were calculated using the $\mathrm{FCH}$ approximation, a broadening parameter of $0.7 \mathrm{eV}$ and a $4 \times 4 \times 4$ sampling of the supercell Brillouin zone. Because of the experimental conditions (see Fig. 1), two types of theoretical spectra were calculated along the following procedure: dipole (E1) and quadrupole (E2) contributions (see Eq. 4) were calculated for two $(\hat{\boldsymbol{\varepsilon}}, \boldsymbol{k})$ couples yielding, respectively, the maximum and the minimum of the pre-edge first peak intensity. These two components were then averaged to provide a E2 in-plane mean spectrum whereas their difference was directly associated with the E2 in-plane dichroic signal. Converged spectra were then obtained by averaging the contributions arising from 40 atomic configurations, considering for each configuration the two $\mathrm{Ti}$ sites of the conventional primitive cell to host the absorbing atom (labeled Ti1 and Ti2 in Fig. 1). As for the $\alpha-\mathrm{Al}_{2} \mathrm{O}_{3}$ case, individual spectra were core-level shifted with respect to each other using $\triangle \mathrm{SCF}$ calculations. ${ }^{54}$ Finally the converged spectra were aligned with experiment in such a way that the A3 pre-edge peak of the $300 \mathrm{~K}$ calculated spectrum matches the corresponding experimental structure.

An additional difficulty, inherent to the method employed to account for thermal fluctuations, arises however in the latter case. As proved in Appendix A, thermal effects and vibrations do not modify the angular dependence of the XAS cross section as imposed by the point group of the crystal. Thus, rutile $\mathrm{TiO}_{2}$ retains its $\mathrm{D}_{4 \mathrm{~h}}$ point group symmetry over the entire temperature range 
investigated in this study. However, individual atomic configurations generated to calculate the spectra do not display the full crystal symmetry any more and in general, do not display any point symmetry at all. Given the small amplitude of the displacements from equilibrium positions introduced in the supercells, the overall loss of symmetry barely affects the shape of the E2 in-plane mean spectra. Its effect is however much more visible on the E2 in-plane dichroic signal. As mentioned above, under our experimental conditions, one expects an absence of electric dipole (E1) contribution to the dichroic signal which is not verified in the numerical calculations where a residual contribution remains present. Attempts to converge this residual E1 signal to zero, i.e. to restore the crystal symmetry by averaging a sufficient number of configurations, proved to be out of reach. An alternative approach to solve this issue is to retrieve the high symmetry component of the absorption cross section from calculations performed for a set of distinct $(\hat{\boldsymbol{\varepsilon}}, \boldsymbol{k})$ couples within the same atomic configuration. A detailed and self-contained presentation of the approach followed in this work is given in Appendix B.

\section{RESULTS AND DISCUSSION}

\section{A. XAS at the Ti $K$ edge in rutile $\mathrm{TiO}_{2}$}

\section{Comparison between calculated and measured spectra}

Fig. 2 shows a comparison between measured and calculated E2 in-plane mean Ti $K$ edge XAS spectra in rutile $\mathrm{TiO}_{2}$ for three different temperatures, using the theoretical approach described in Sec. IIC. The E2 inplane mean pre-edge region is presented in more details in Fig. 3, where the E1 and E2 contributions to the calculated spectra are shown separately. Finally, a comparison between the theoretical and the experimental inplane dichroic signals in the same pre-edge energy range is shown in Fig. 4. As mentioned above, this dichroic signal was obtained as the difference between the spectra recorded for an in-plane orientation of the polarization yielding the highest and lowest pre-edge first peak intensities, respectively.

The pre-edge region is essentially built from three peaks, labeled A1, A2 and A3 in the figures, arising at $4966,4968.5$ and $4972 \mathrm{eV}$, respectively, while the main edge region is dominated at higher energies by a clear shoulder labeled B at $4978 \mathrm{eV}$, a faint feature labeled $\mathrm{C}$ at $4981.5 \mathrm{eV}$ and a major peak labeled $\mathrm{D}$ at about $4984 \mathrm{eV}$. All these observations are consistent with the spectra already published in the literature on this compound. ${ }^{39,56-63}$ This spectrum shows a clear evolution as temperature increases. In the pre-edge region, intensities of peaks A1 and A2 are both increasing with temperature while peak A3 remains virtually unchanged. In the main edge region, peak $\mathrm{D}$ experiences the largest intensity variation, decreasing strongly with increasing

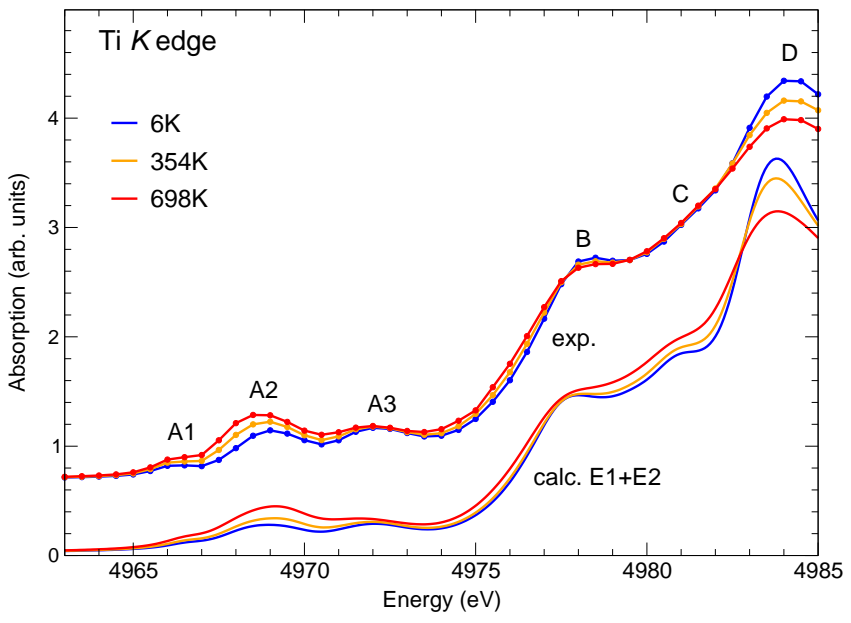

FIG. 2: Comparison between the experimental and the calculated E2 in-plane mean XAS spectra at the Ti $K$ edge in rutile $\mathrm{TiO}_{2}$, for three different temperatures. The experimental spectra (full lines with data points) were shifted vertically for clarity.

temperatures. Peak B follows the same trend with, however, a much smaller amplitude while $\mathrm{C}$ remains essentially unchanged. It should also be noted that peak A2 shifts by about $0.5 \mathrm{eV}$ towards lower energies when increasing the temperature from 6 to $698 \mathrm{~K}$.

The experimental in-plane dichroic signal (Fig. 4) arises mostly in the energy range corresponding to the first two peaks of the pre-edge region, changing sign from positive to negative when moving from A1 to A2, and weakens slightly as temperature increases from 6 to $698 \mathrm{~K}$.

The Ti $K$ edge spectra (Fig. 2) were calculated for three different temperatures employed in the experiments. They all exhibit a similar shape as the experimental spectra. In the pre-edge region, A2 and A3 peaks are rather well reproduced regarding their relative intensity and energy position. Peak A1 is well reproduced as far as its relative intensity with respect to the other preedge features is concerned, but occurs at slightly higher energy than in the experiments. The main edge features are also well reproduced regarding their relative energy position and intensity, though feature $\mathrm{C}$ is slightly too intense compared to the experiments and is affected by temperature. Nevetheless, the temperature dependence of the spectra also shows an overall good agreement with the experiments. In particular, the intensity increase of peaks A1 and A2 and decrease of peak D with increasing temperature is correctly predicted. In the pre-edge region, the main difference between theory and experiments lies, however, in the absence of the temperature-induced energy shift of the A2 pre-edge peak in the calculations.

The E2 in-plane dichroic signal is also overall well reproduced although the calculated signal is slightly shifted towards higher energies (see Fig. 4). As observed in the 


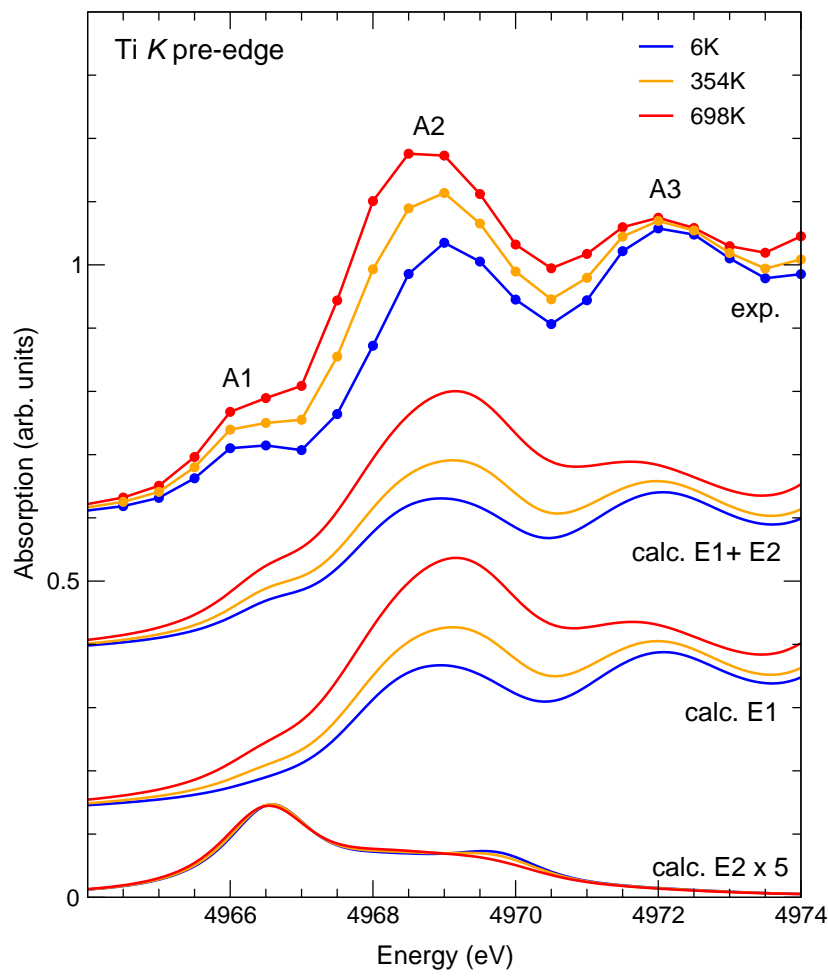

FIG. 3: Temperature dependence of the Ti $K$ pre-edge region in rutile $\mathrm{TiO}_{2}$. Calculated E1 and E2 contributions (solid lines), as well as their sum, are compared to experiment (solid lines with data points) for three different temperatures. Spectra were vertically shifted for clarity.

experiments, the dichroism is mainly present at the A1 and A2 pre-edge peaks and arises exclusively from the E2 contribution. The temperature dependence of the signal (and thus of the anisotropy in the rutile crystal) is also well reproduced, as it decreases slightly with increasing temperatures, in agreement with the experimental data.

Overall, Figs. 2, 3 and 4 show how the present method proves to be essential to grasp the effect of temperature on the spectral shape and represents a real improvement over a standard calculation, where atoms are set to their equilibrium position and where temperature is accounted for, at most, through the thermal expansion of lattice parameters.

\section{Pre-edge region analysis}

Pre-edge structures in XAS give insight into the local atomic and electronic structures of the absorbing atom. More specifically, in the case of transition metals, the $\mathrm{K}$ pre-edge region provides a direct access to the manifold of empty $d$ states. As shown in Fig. 3, the pre-edge peaks are here the result of electric dipole, electric quadrupole, or of a combination of both types of electronic transitions. More precisely, both E1 and E2 transitions con-

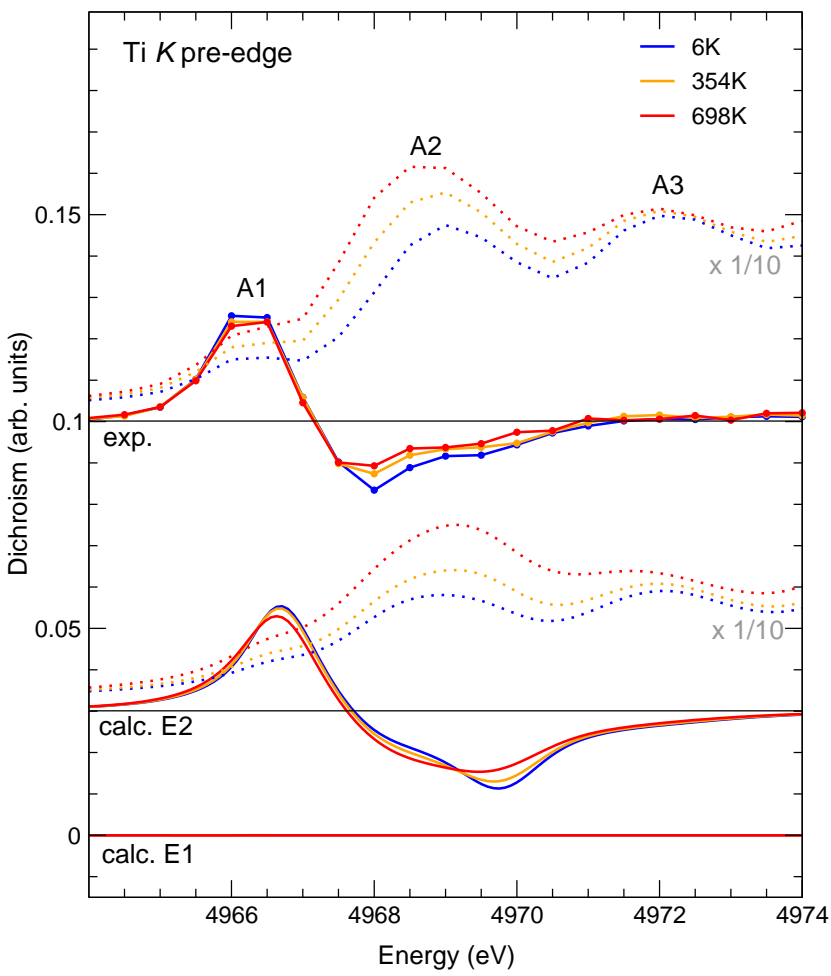

FIG. 4: Experimental and calculated in-plane dichroic signal of the $\mathrm{Ti} K$ pre-edge region in rutile $\mathrm{TiO}_{2}$ for three different temperatures. The intensity of the dichroic signal is in the same units as those used for the XAS spectra in Fig. 3. Zero lines (in black) have been added for measured and calculated dichroism, which have been vertically shifted for clarity. For comparison, the XAS spectra are also displayed in dotted lines, but using a factor $1 / 10$.

tribute to A1 and A2 peaks while A3 arises solely from E1 transitions. In other words, both $\mathrm{A} 1$ and $\mathrm{A} 2$ peaks originate from $1 s \rightarrow p$ and $1 s \rightarrow d$ transitions while A3 arises purely from $1 s \rightarrow p$ transitions. This assignment is valid for all the temperatures investigated.

Moreover, E1 transitions possess either a local or a non-local character. Local transitions involve transitions to the absorbing atom $p$ states hybridized with the absorbing atom $d$ states, whereas non-local ones involve transitions to the absorbing atom $p$ states mixed with the neighbour cations $d$ states. This is important here because the point group of the $\mathrm{Ti}$ site in rutile $\mathrm{TiO}_{2}$ $\left(\mathrm{D}_{2 \mathrm{~h}}\right)$ is centrosymmetric. In this case, on-site $p$ - $d$ mixing is symmetry-forbidden and only non-local E1 transitions should be visible in the pre-edge region. However, taking vibrations into account breaks the local symmetry and relaxes the above-mentioned constraint, allowing local $p$ - $d$ hybridization to take place. The presence of local E1 transitions in the pre-edge region is thus enabled by vibrations.

This mechanism explains the presence of E1 contributions to peak A1, which would otherwise be symmetryforbidden. Fig. 5 compares the pre-edge region of the 


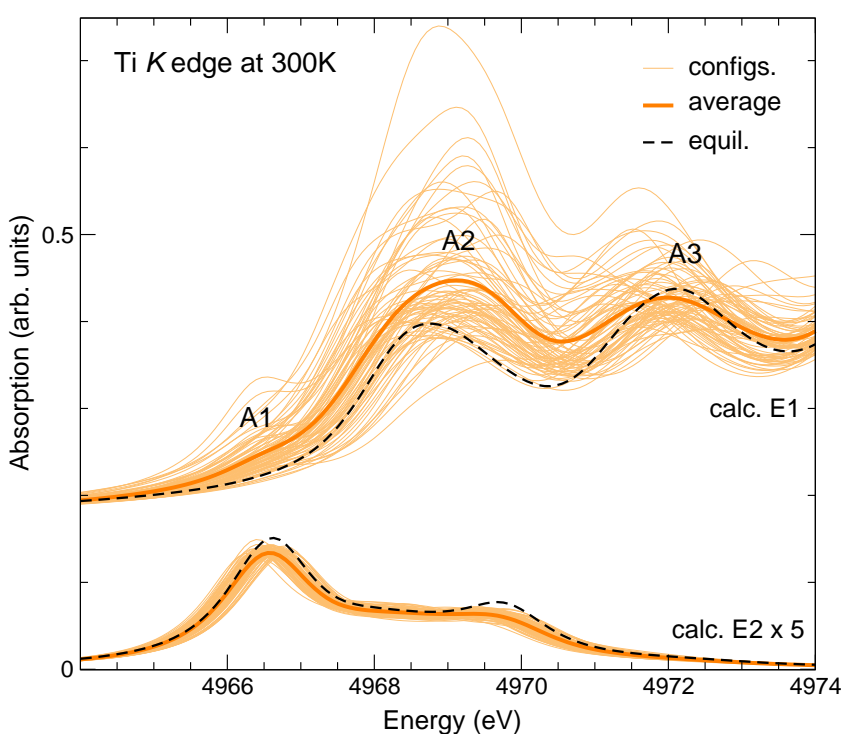

FIG. 5: Theoretical Ti $K$ pre-edge structures in rutile $\mathrm{TiO}_{2}$ at 300K: comparison between spectra performed with atoms at equilibrium positions (black dashed line) and obtained when taking vibrations into account (orange thick line), which results from the average of the configuration spectra (light orange thin lines). The E1 and E2 contributions are shown separately and shifted vertically for clarity.

Ti $K$ edge calculated at $300 \mathrm{~K}$ when vibrations are taken into account (orange lines) or neglected (dashed black lines). Fig. 5 indicates that the spectrum only shows an E1 contribution to the A1 peak if vibrations are taken into account. Another sizeable effect of atomic vibrations on the E1 contribution is observed for the A2 pre-edge structure and, to a much weaker extent, for the A3 peak. On the contrary, temperature effects leave the E2 contribution almost unaffected save for a slight broadening of the structures.

Inspection of the projected densities of states (PDOS) presented in Fig. 6 confirms these results. The top panel of the figure shows a comparison between the spectrum calculated with atoms at their equilibrium positions, together with individual E1 and E2 contributions and the experimental spectrum. Lower panels show, from top to bottom, PDOS projected on the absorbing Ti $p$, neighboring Ti $p$, absorbing Ti $3 d$ and neighboring Ti $3 d$. A decomposition of the Ti $3 d$ states is performed according to the symmetry properties of the $3 d$ orbitals, i.e. onto the irreducible representations of the Ti site point group. In $\mathrm{D}_{2 \mathrm{~h}}$ symmetry, the $d$ manifold of the transition metal ion is split into non-degenerate $\mathrm{A}_{\mathrm{g}}, \mathrm{B}_{2 \mathrm{~g}}, \mathrm{~B}_{3 \mathrm{~g}}$ states which will be referred to as " $t_{2 g}$-like" orbitals in the reminder by reference to their properties in octahedral symmetry and into $\mathrm{A}_{\mathrm{g}}$ and $\mathrm{B}_{1 \mathrm{~g}}$ states for the " $e_{g}$-like" orbitals. ${ }^{64}$ The local system of coordinates employed in these calculations is shown in the inset of Fig. 6.

Fig. 6 illustrates how, at equilibrium, the only possible contribution to A1 arises from local E2 transitions.

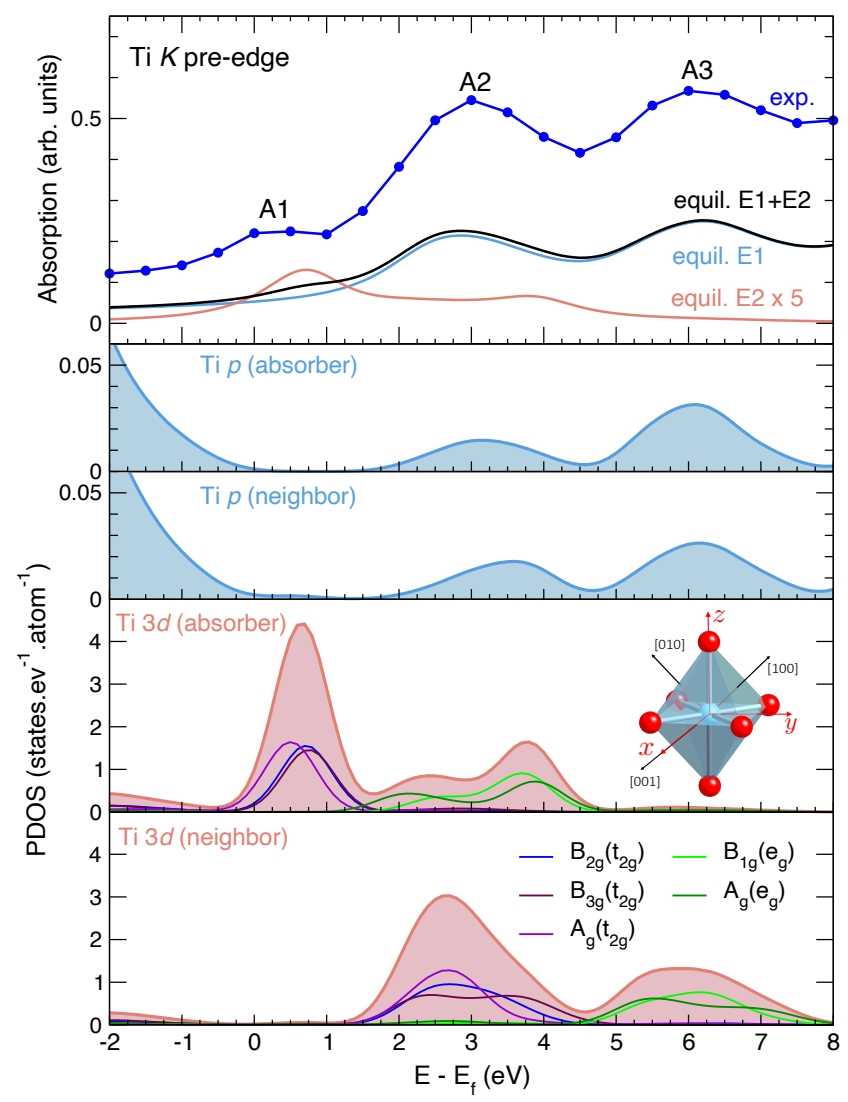

FIG. 6: Top panel: experimental (6K) and and calculated Ti $K$ in-plane mean pre-edge XAS spectra in rutile. Panels below: partial $p$ and $3 d$ densities of states projected on the absorbing $\mathrm{Ti}$ and one of its first $\mathrm{Ti}$ neigbor. The supercell used for XAS and PDOS calculations was built with atoms at their equilibrium positions using the LDA-optimized crystal structure.

Indeed, only the absorbing Ti $t_{2 g}$-like empty orbitals are located in this energy range. This is in agreement with most of previous studies. ${ }^{32,39,56-62}$ Note that the attribution of this peak to dipole transitions made by $\mathrm{Wu}$ et $a l .{ }^{63}$ and Brydson et $a l .{ }^{65}$ was seemingly due to artifacts introduced by the muffin-tin approximation. ${ }^{39}$ Moreover, Fig. 6 shows the mixed character of A2, which stems from both non-local E1 and local E2 contributions at equilibrium. PDOS indeed confirm that these components are consistent with the presence of, respectively, absorbing $\mathrm{Ti} 4 p$ states hybridized with neighboring $\mathrm{Ti} t_{2 g}$-like and absorbing Ti $e_{g}$-like states in this energy range. Finally, PDOS also show that the only electronic states at the origin of A3 are the $e_{g}$-like states of neigboring cations, hybridized with absorbing $\mathrm{Ti} 4 p$ states. This thus confirms the purely non-local character of this peak. A summary of the electronic transitions occurring at the Ti K pre-edge structures is given in Tab. I.

The effect of temperature on the pre-edge structures can therefore be understood as follows. As the thermal motion of the nuclei barely affects E2 transitions (see 


\begin{tabular}{lll}
\hline \hline Peak labels & Probed states & Electronic transitions \\
\hline A1 & $\begin{array}{l}\text { absorbing Ti } 3 d\left(t_{2 g} \text {-like }\right) \\
\text { absorbing Ti } 3 d\left(t_{2 g} \text {-like }\right)\end{array}$ & $\begin{array}{l}\text { E2: } 1 s \rightarrow 3 d \\
\text { E1 local }: 1 s \rightarrow 4 p \text { mixed with } 3 d \text { of absorbing Ti }\end{array}$ \\
& $\begin{array}{l}\text { absorbing Ti } 3 d\left(e_{g} \text {-like }\right) \\
\text { Absorbing Ti } 3 d\left(e_{g} \text {-like }\right)\end{array}$ & E2: $1 s \rightarrow 3 d$ \\
& E1 local $: 1 s \rightarrow 4 p$ mixed with $3 d$ of absorbing Ti \\
& neighboring Ti $3 d\left(t_{2 g}\right.$-like $)$ & E1 non-local $: 1 s \rightarrow 4 p$ mixed with $3 d$ of neighboring Ti \\
A3 & E1 non-local $: 1 s \rightarrow 4 p$ mixed with $3 d$ of neighboring Ti \\
\hline \hline
\end{tabular}

TABLE I: Nature of the electronic transitions at the origin of the Ti $K$ pre-edge structures in rutile $\mathrm{TiO}_{2}$. Transitions induced by nuclear thermal fluctuations are indicated in bold.

bottom of Fig. 5), the intensity variation of peak A1 is entirely due to temperature-induced local E1 contributions (see top of Fig. 5). The relatively weak amplitude of this contribution compared to the dominant E2 signal in this energy range and the predicted absence of on-site $p$ - $d$ hybridization explain why it has often been overlooked in previous works on rutile $\mathrm{TiO}_{2}$. A recent indepth study ${ }^{66}$ of the linear dichroism of this edge, based on high-energy-resolution measurements, confirms however the presence of a weak E1 contribution to this peak, although the authors did not relate it explicitely to lattice vibrations. The most important effects, observed on the A2 peak, originate from the same vibration-induced local E1 transitions, but in this case towards the $e_{g}$-like orbitals of the absorber. It is interesting to note that this strong temperature dependence for transitions to $e_{g^{-}}$ related final states is not restricted to rutile $\mathrm{TiO}_{2}$ but also observed in other compounds such as $\mathrm{SrTiO}_{3}{ }^{67}$ or $\mathrm{PbTiO}_{3}{ }^{10}$

As mentioned above, the slight redshift of peak A2 with increasing temperature is not properly reproduced in the calculations. A qualitative explanation of this result lies in the approximate treatment of the core-hole provided by DFT. Indeed, the PDOS shown in Fig. 6 illustrate the strong effect of the locally more attractive potential of the absorber on its unoccupied $3 d$ orbitals, pulling these states down in energy by a few eV. However, DFT has a tendency to overestimate the screening of the $1 s$ core-hole by the valence electron density, which appears as the main drawback of this method. ${ }^{32,68}$ The resulting misplacement of the absorber $3 d$ orbitals is thus directly echoed in a temperature-induced local E1 contribution located at about the same energy as the nonlocal contribution, leading to an overall energy position of this structure independent of the temperature. A more attractive core-hole potential would lower the energy of these localized $3 d$ states, which in turn would lower the energy of the local E1 contribution and restore the redshift of peak A2 observed experimentally as temperature increases.

We close this discussion of the $\mathrm{Ti} K$ edge in rutile $\mathrm{TiO}_{2}$ with a few remarks on the temperature-dependence of the E2 in-plane dichroic signal. As detailed in Appendix B, a proper calculation of this signal when accounting for finite temperature effects with the present method requires the explicit cancellation of additional terms arising in the cross section as symmetry is lost through the random displacement of atoms in the supercell. The present work indeed shows that the crystal symmetry cannot be restored simply by averaging over a reasonable number of configurations. Extraction of the high-symmetry components of the cross section, corresponding to the full symmetry of the crystal at equilibrium, proves however very efficient and generally applicable. As shown in Fig. 4, this leads to a complete cancellation of the E1 in-plane dichroic component and to a fast convergence of the E2 signal with the number of configurations, comparable to the convergence achieved for the in-plane mean spectra. The accurate reproduction of the subtle temperature-dependence of this signal confirms the relevance of this approach which adds, therefore, a new dimension to the methodology developed here to account for thermal effects on core-level excitation spectra.

\section{B. XRS at the Al $K$ edge in $\alpha-\mathrm{Al}_{2} \mathrm{O}_{3}$}

The top of Fig. 7 compares experimental and theoretical $\mathrm{Al} K$ edge XRS spectra in $\alpha-\mathrm{Al}_{2} \mathrm{O}_{3}$ for a module of the scattering vector $q=10 \AA^{-1}$. The two main spectral features visible in the experimental data consist in a preedge peak labeled $\mathrm{A}$ in the figure, arising at $1564 \mathrm{eV}$ and a main edge peak labeled $\mathrm{B}$ at $1567 \mathrm{eV}$. The theoretical spectra, obtained by a standard calculation where all atoms are fixed to their equilibrium positions (black solid line) and by taking vibrations at room temperature into account (orange solid line), both reproduce the overall shape of the experimental one. The explicit account of finite temperature effects in the calculations clearly improves the agreement with experiment by reducing the intensity of peak B, which is largely overestimated when considering the equilibrium atomic structure only. The description of damped structures arising above $1568 \mathrm{eV}$ is also improved in the finite temperature calculations.

The calculated dynamic structure factor is the sum of two transition-channel contributions that are shown in 


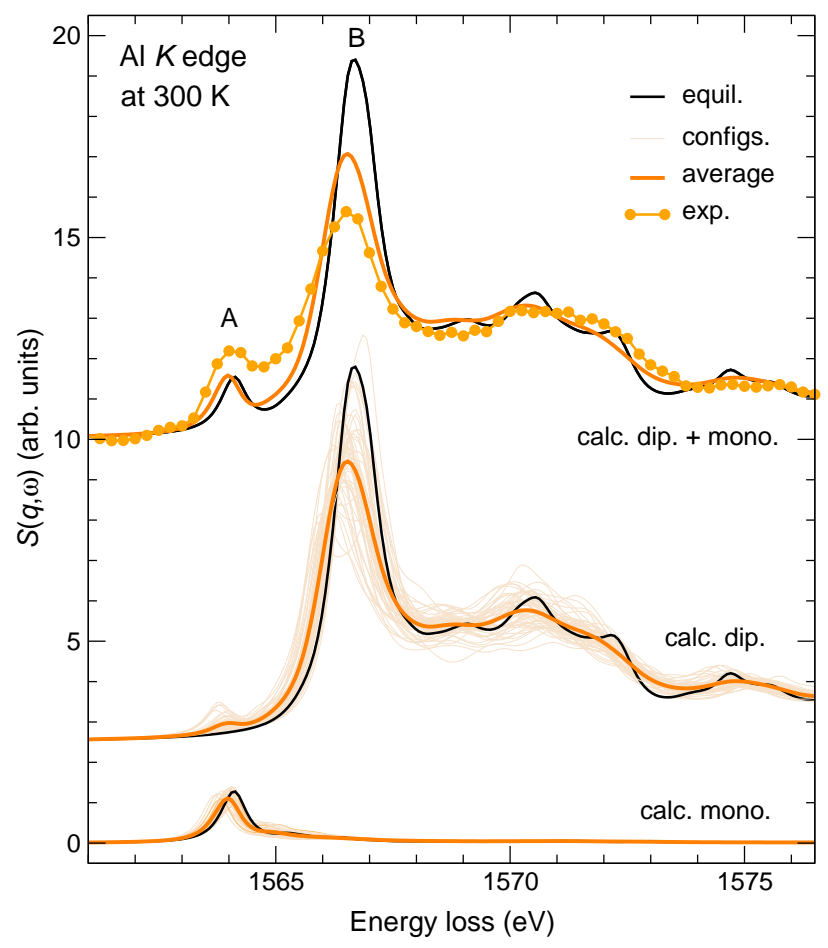

FIG. 7: $\mathrm{Al} K$-edge XRS spectra in corundum $\alpha-\mathrm{Al}_{2} \mathrm{O}_{3}$ at $300 \mathrm{~K}$, for momentum transfer $q=10 \AA^{-1}$ : comparison between experiment and calculations performed with atoms at equilibrium positions (black solid line) and obtained when taking vibrations into account (orange thick line), which results from the average of the configuration spectra (light orange thin lines). The monopole and dipole contributions are shown separately and shifted vertically for clarity.

the middle and bottom of Fig. 7. Peak A mainly results from $1 s \rightarrow 3 s$ monopole transitions while higherenergy structures are due to $1 s \rightarrow p$ dipole transitions. For both monopole and dipole contributions, the configuration spectra are displayed. While the monopole dynamic structure factor is only slightly affected by vibrations (shift towards lower energy of $0.1 \mathrm{eV}$ and intensity damping of about 14\%), the dipole one concentrates the main effects described above, with peak B largely reduced and high-lying structures broadened. More strikingly, vibrations give rise to a weak dipole contribution in the pre-edge region. Similarly to the previous case of $\mathrm{TiO}_{2}$, temperature-induced atomic displacements lead to an enhanced on-site, or local hybridization, mixing here the absorber $s$ and $p$ unoccupied states. The pre-edge peak A results therefore from both electric monopole and dipole transitions.

In XRS, the $K$ pre-edge region of light elements, such as $\mathrm{Al}$, is indeed particularly interesting as it concentrates most of the multipolar transitions which can be observed for such edges with this spectroscopy. ${ }^{35}$ More specifically, monopole contributions, resulting from $1 s \rightarrow 3 s$ electronic transitions, are strongly enhanced when large mo-

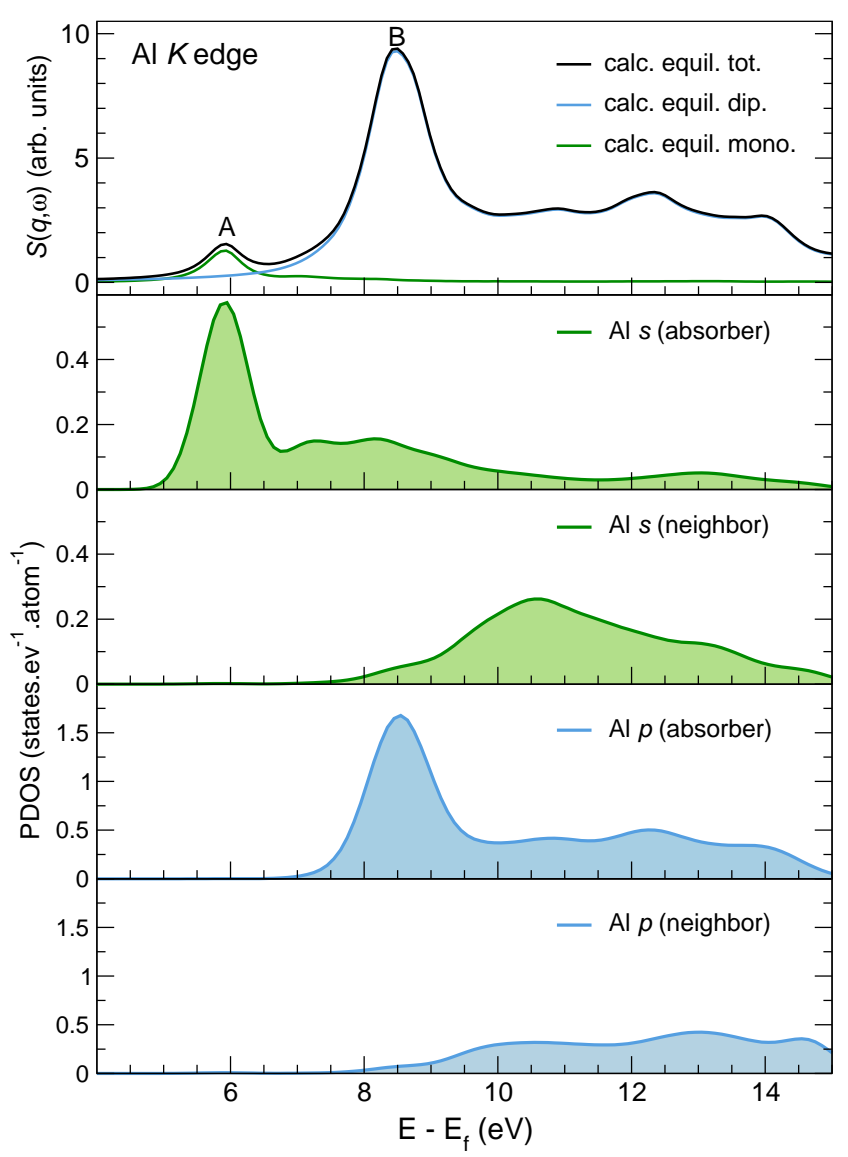

FIG. 8: Top panel: theoretical XRS spectra at the Al $K$-edge in $\alpha-\mathrm{Al}_{2} \mathrm{O}_{3}$, calculated for $q=10 \AA^{-1}$ and decomposed into monopole and dipole contributions. Panels below: partial $s$ and $p$ densities of states projected on absorber $\mathrm{Al}$ and one of its first Al neigbor. The supercell used for $S(\boldsymbol{q}, \omega)$ and PDOS calculations was built with atoms at their equilibrium positions using the experimental crystal structure at $300 \mathrm{~K} .^{53}$

menta are transferred, giving rise in our case (see Fig. 7) to the largest contribution to peak A. In Fig. 8, the PDOS calculated for the structure at equilibrium confirm this picture. The bottom of the conduction band is purely built from the absorber $3 s$ states hybridized with ligand $p$ states. The absorber $p$ states only appear at about $3 \mathrm{eV}$ higher in energy. Besides, the PDOS shown for both excited (absorber) and non excited (neighbor) Al clearly evidence the strong effect of the core-hole on both $s$ and $p$ unoccupied states, at the origin of peaks $\mathrm{A}$ and $\mathrm{B}$ in the equilibrium total spectrum, respectively.

\section{CONCLUSION}

In this work, we employed an approach based on density-functional theory to assess the effect of lattice quantum dynamics on two different core-level spectroscopies allowing for multipole electronic transitions: XAS 
at the $\mathrm{Ti} K$ edge in rutile $\mathrm{TiO}_{2}$ where electric dipole and quadrupole transitions dominate the pre-edge region and XRS at the $\mathrm{Al} K$ edge in $\alpha-\mathrm{Al}_{2} \mathrm{O}_{3}$ where monopole and dipole transitions are observed for large scattering vectors. In both cases, accounting for thermal effects clearly improves the agreement between experiments and theory. Indeed, besides the overall broadening of the spectral features, a strong effect of the temperature is observed on the dipole allowed electronic transitions, leading either to an enhancement of the spectral weight of certain features already visible in the calculations performed at equilibrium or to the appearance of new electronic transitions.

These effects essentially originate from on-site hybridization between the absorber $p$ and $s$ or $d$ localized unoccupied states arising when atomic nuclei are displaced from their equilibrium positions and occur, therefore, in energy regions dominated by these latter states. On the contrary, monopole and quadrupole transitions to final $\mathrm{Al} 3 s$ states in XRS and Ti $3 d$ states in XAS, respectively, prove to be fairly insensitive to temperature, whose dominant effect is reduced to a slight broadening of the structures. Although difficult to extrapolate to other systems than the oxides under study, our results show that the higher sensitivity to local atomic displacements of the absorber unoccupied $p$ states is related to their larger spatial extension and higher energy position. The absorber $\mathrm{Al} 3 s$ or $\mathrm{Ti} 3 d$ states experience a stronger localization in the presence of a $1 s$ core-hole leading to a large redshift of these states visible on the PDOS, toward the bottom of the conduction band. These states therefore tend to decouple from the continuum of the conduction states, reducing considerably their sensitivity to the details of the surrounding environment.

Finally, we proposed a general method devised to properly account for the orientation dependence of core-level spectroscopies in calculations performed at finite temperatures. This method, based on a group-theoretical analysis, consists in cancelling all spurious contributions to the absorption or scattering cross sections, which arise from the artificial loss of the crystal symmetry in the atomic configurations generated to account for lattice quantum dynamics. The good agreement between theory and experiments obtained on the temperature dependent dichroic signal of the Ti $K$ pre-edge region in rutile $\mathrm{TiO}_{2}$ demonstrates the relevance of this approach. As this method enables to rigorously treat angular dependence at finite temperature, it can be extended to the calculation of any natural dichroism in systems studied in non-equilibrium configurations.

\section{Acknowledgments}

This work was granted access to the HPC resources of IDRIS under the allocation 2018-A0040906863, 2019A0060906863 and 2020-A0080906863 made by GENCI. S.H. was supported by Academy of Finland (grant 1295696). We thank ESRF and the Diamond Light
Source for beamtime. S.D. acknowledges Emmanuelle de Clermont Gallerande for her kind support in XRS calculations.

\section{APPENDIX A}

In the present study, theoretical spectra are obtained as an average of cross sections calculated for out-ofequilibrium atomic configurations of the crystalline material, by using the framework described in Ref 30 . Because in each of those configurations, atoms are displaced from their equilibrium positions, the symmetry properties of the crystal are lost, leading to an angular dependence of the average cross section different from that obtained with atoms at their equilibrium positions. In this Appendix, we show that even under the effect of temperature-induced harmonic vibrations, the angular dependence of XAS (or XRS) spectra has to be that imposed by the symmetry point group of the crystal. In other words, an atomic displacement in one direction is equiprobable to a displacement in another direction obtained by the symmetry operations of the crystallographic point group. To be consistent with the Appendix of Ref. 30, the demonstration below is done in a multielectronic scheme within the Born-Oppenheimer approximation.

First of all, we recall the main theoretical ingredients of the method of Nemausat et al. ${ }^{30}$ used in this paper.

The crystal is seen as a system of $N$ nuclei of mass $M_{I}$ and charge $q_{I}(I=1, \ldots, N)$ and $N_{e}$ electrons of mass $m$, with respective position vectors $\left(\boldsymbol{R}_{1}, \ldots, \boldsymbol{R}_{N}\right)$ and $\left(\boldsymbol{r}_{1}, \ldots, \boldsymbol{r}_{N_{e}}\right)$. The system is described by wavefunctions $\Psi(\overline{\boldsymbol{R}}, \overline{\boldsymbol{r}})$ of energy $E$, where we have used collective coordinates $\overline{\boldsymbol{R}}=\left(\boldsymbol{R}_{1}, \ldots, \boldsymbol{R}_{N}\right)$ and $\overline{\boldsymbol{r}}=\left(\boldsymbol{r}_{1}, \ldots, \boldsymbol{r}_{N_{e}}\right)$ to simplify the notation.

According to Ref. 30, for each incoming-photon energy $\hbar \omega$, the XAS cross section including vibrations at temperature $T$ can be written as:

$$
\begin{aligned}
\sigma(\hbar \omega)= & 4 \pi^{2} \alpha_{0} \hbar \omega \frac{1}{Z} \sum_{m, n, i, j} e^{-E_{m}^{i} / k_{\mathrm{B}} T} \\
& \times\left|\left\langle\Psi_{n}^{j}|\mathcal{O}(\overline{\boldsymbol{r}})| \Psi_{m}^{i}\right\rangle\right|^{2} \delta\left(E_{n}^{j}-E_{m}^{i}-\hbar \omega\right)(\mathrm{A} 1)
\end{aligned}
$$

where $\alpha_{0}$ and $k_{\mathrm{B}}$ are the fine structure constant and the Boltzmann constant, respectively, and where the partition function reads

$$
Z=\sum_{m, i} e^{-E_{m}^{i} / k_{\mathrm{B}} T}
$$

In Eq. $\mathrm{A} 1, \mathcal{O}(\overline{\boldsymbol{r}})$ is the XAS transition operator,

$$
\mathcal{O}(\overline{\boldsymbol{r}})=\sum_{j} e^{\mathrm{i} \boldsymbol{k} \cdot \boldsymbol{r}_{j}}\left(\hbar \hat{\boldsymbol{\varepsilon}} \cdot \nabla_{j}-\frac{g}{2} \boldsymbol{s}_{j} \cdot(\boldsymbol{k} \times \hat{\boldsymbol{\varepsilon}})\right)
$$

that, after the multipole expansion, leads to the E1 and E2 operators given by Eq. 2 and Eq. 3 in a single-electron 
scheme, respectively. In our method, wavefunctions $\Psi_{m}^{i}(\overline{\boldsymbol{r}}, \overline{\boldsymbol{R}})$ are described within the Born-Oppenheimer approximation and using the harmonic approximation.

In the Born-Oppenheimer approximation, we assume that wavefunctions $\Psi(\overline{\boldsymbol{r}}, \overline{\boldsymbol{R}})$ can be factorized

$$
\Psi_{m}^{i}(\overline{\boldsymbol{r}}, \overline{\boldsymbol{R}})=\chi_{m}^{i}(\overline{\boldsymbol{R}}) \psi_{m}(\overline{\boldsymbol{r}} ; \overline{\boldsymbol{R}}) .
$$

In Eq. A4, electronic wavefunction $\psi_{m}(\overline{\boldsymbol{r}} ; \overline{\boldsymbol{R}})$ is the solution of Schrödinger equation with nuclei clamped at position $\overline{\boldsymbol{R}}$

$$
H_{e}(\overline{\boldsymbol{r}} ; \overline{\boldsymbol{R}}) \psi_{m}(\overline{\boldsymbol{r}} ; \overline{\boldsymbol{R}})=\epsilon_{m}(\overline{\boldsymbol{R}}) \psi_{m}(\overline{\boldsymbol{r}} ; \overline{\boldsymbol{R}}),
$$

where the electronic Hamiltonian reads

$H_{e}(\overline{\boldsymbol{r}} ; \overline{\boldsymbol{R}})=-\frac{\hbar^{2}}{2 m} \sum_{i=1}^{N_{e}} \Delta_{i}+V_{e}(\overline{\boldsymbol{r}})+V_{e N}(\overline{\boldsymbol{r}}, \overline{\boldsymbol{R}})+V_{N}(\overline{\boldsymbol{R}})$,

$V_{e}$ being the Coulomb potential between electrons, $V_{e N}$ the Coulomb potential between nuclei and electrons and $V_{N}$ the Coulomb potential between nuclei.

In the harmonic approximation, the vibrational wavefunction $\chi_{m}^{i}(\overline{\boldsymbol{R}})($ Eq. A4) is the solution of

$$
H_{m}\left(\overline{\boldsymbol{R}}, \overline{\boldsymbol{R}}^{0}\right) \chi_{m}^{i}(\overline{\boldsymbol{R}})=\left(E_{m}^{i}-\epsilon_{m}\left(\overline{\boldsymbol{R}}^{0}\right)\right) \chi_{m}^{i}(\overline{\boldsymbol{R}}),
$$

where $\overline{\boldsymbol{R}}^{0}$ are the equilibrium positions of the nuclei for quantum state $\psi_{m}$ and with

$$
\begin{aligned}
H_{m}\left(\overline{\boldsymbol{R}}, \overline{\boldsymbol{R}}^{0}\right) & =-\sum_{I=1}^{N} \frac{\hbar^{2}}{2 M_{I}} \Delta_{\boldsymbol{R}_{I}} \\
& +\left.\frac{1}{2} \sum_{I, J}\left(\boldsymbol{R}_{I}-\boldsymbol{R}_{I}^{0}\right) \cdot \frac{\partial^{2} \epsilon_{m}(\overline{\boldsymbol{R}})}{\partial \boldsymbol{R}_{I} \partial \boldsymbol{R}_{J}}\right|_{\overline{\boldsymbol{R}}=\overline{\boldsymbol{R}}^{0}}
\end{aligned}
$$

If we assume that the experimental resolution is much larger than the vibrational energies, ${ }^{23}$ we can neglect the final state vibrations and we concentrate on the vibrational energies of the ground state given by the eigenvalues of $H_{m}$. In other words, Eq. A6 is solved for $m=0$.

Within this formalism, the $\sigma(\hbar \omega)$ cross section including temperature and vibrations (Eq. A1) becomes an average over configuration cross sections $\sigma_{\overline{\boldsymbol{R}}}(\hbar \omega):{ }^{30}$

$$
\begin{aligned}
\sigma_{\mathrm{av}}(\hbar \omega)= & Z^{-1} \sum_{i} e^{-E_{0}^{i} / k_{\mathrm{B}} T} \\
& \times \int d \overline{\boldsymbol{R}}\left|\chi_{0}^{i}(\overline{\boldsymbol{R}})\right|^{2} \sigma_{\overline{\boldsymbol{R}}}(\hbar \omega),
\end{aligned}
$$

with

$\sigma_{\overline{\boldsymbol{R}}}(\hbar \omega)=4 \pi^{2} \alpha_{0} \hbar \omega \sum_{f}\left|\left\langle\psi_{f}|\mathcal{O}(\overline{\boldsymbol{r}})| \psi_{0}\right\rangle\right|^{2} \delta\left(\epsilon_{f}-\epsilon_{0}-\hbar \omega\right)$.

Now, we consider an arbitrary isometry $D$, which can always be written as $D \boldsymbol{r}=\mathcal{S} \boldsymbol{r}+\boldsymbol{t}$ for any $\boldsymbol{r}$, and where $\boldsymbol{t}$ is a translation vector and $\mathcal{S}$ a rotation or a roto-inversion. By changing variable $\overline{\boldsymbol{R}}$ to $D \overline{\boldsymbol{R}}$ we obtain

$$
\begin{aligned}
\sigma_{\mathrm{av}}(\hbar \omega)= & Z^{-1} \sum_{i} e^{-E_{0}^{i} / k_{\mathrm{B}} T} \\
& \times \int d \overline{\boldsymbol{R}}\left|\chi_{0}^{i}(D \overline{\boldsymbol{R}})\right|^{2} \sigma_{D \overline{\boldsymbol{R}}}(\hbar \omega),
\end{aligned}
$$

where we used $d D \overline{\boldsymbol{R}}=d \overline{\boldsymbol{R}}$ because isometries conserve volume.

First, we investigate the $\sigma_{\overline{\boldsymbol{R}}}(\hbar \omega)$ configuration cross section when $D$ is applied to $\overline{\boldsymbol{R}}$. Since isometries do not change length and scalar product and since the $V_{e N}(\overline{\boldsymbol{r}}, \overline{\boldsymbol{R}})$ and $V_{N}(\overline{\boldsymbol{R}})$ Coulomb terms of Eq. A5 are functions of length of type $\left|\boldsymbol{r}_{i}-\boldsymbol{R}_{I}\right|$ and $\left|\boldsymbol{R}_{I}-\boldsymbol{R}_{J}\right|$, respectively, we can easily show that

$$
H_{e}(\overline{\boldsymbol{r}} ; D \overline{\boldsymbol{R}})=H_{e}\left(D^{-1} \overline{\boldsymbol{r}} ; \overline{\boldsymbol{R}}\right) .
$$

The $\psi_{m}$ electronic wavefunctions satisfy both

$$
H_{e}(\overline{\boldsymbol{r}} ; D \overline{\boldsymbol{R}}) \psi_{m}(\overline{\boldsymbol{r}} ; D \overline{\boldsymbol{R}})=\epsilon_{m}(D \overline{\boldsymbol{R}}) \psi_{m}(\overline{\boldsymbol{r}} ; D \overline{\boldsymbol{R}})
$$

and

$$
H_{e}\left(D^{-1} \overline{\boldsymbol{r}} ; \overline{\boldsymbol{R}}\right) \psi_{m}\left(D^{-1} \overline{\boldsymbol{r}} ; \overline{\boldsymbol{R}}\right)=\epsilon_{m}(\overline{\boldsymbol{R}}) \psi_{m}\left(D^{-1} \overline{\boldsymbol{r}} ; \overline{\boldsymbol{R}}\right) .
$$

Since the two Hamiltonians are identical (Eq. A11), it is always possible to order the eigenvalues (if they are non degenerate) ${ }^{69}$ so that

$$
\epsilon_{m}(D \overline{\boldsymbol{R}})=\epsilon_{m}(\overline{\boldsymbol{R}})
$$

and $\psi_{m}(\overline{\boldsymbol{r}} ; D \overline{\boldsymbol{R}})$ and $\psi_{m}\left(D^{-1} \overline{\boldsymbol{r}} ; \overline{\boldsymbol{R}}\right)$ are equal up to a phase. Then, using these symmetry properties of the electronic states and eigenvalues, we are able to calculate $\sigma_{D \overline{\boldsymbol{R}}}(\hbar \omega)$ starting from Eq. A9. We have

$$
\begin{aligned}
&\left|\left\langle\psi_{f}(\overline{\boldsymbol{r}} ; D \overline{\boldsymbol{R}})|\mathcal{O}(\overline{\boldsymbol{r}})| \psi_{0}(\overline{\boldsymbol{r}} ; D \overline{\boldsymbol{R}})\right\rangle\right|^{2} \\
& \times \quad \delta\left(\epsilon_{f}(D \overline{\boldsymbol{R}})-\epsilon_{0}(D \overline{\boldsymbol{R}})-\hbar \omega\right) \\
&=\quad\left|\left\langle\psi_{f}\left(D^{-1} \overline{\boldsymbol{r}} ; \overline{\boldsymbol{R}}\right)|\mathcal{O}(\overline{\boldsymbol{r}})| \psi_{0}\left(D^{-1} \overline{\boldsymbol{r}} ; \overline{\boldsymbol{R}}\right)\right\rangle\right|^{2} \\
& \quad \times \delta\left(\epsilon_{f}(\overline{\boldsymbol{R}})-\epsilon_{0}(\overline{\boldsymbol{R}})-\hbar \omega\right) \\
&=\quad\left|\left\langle\psi_{f}(\overline{\boldsymbol{r}} ; \overline{\boldsymbol{R}})|\mathcal{O}(D \overline{\boldsymbol{r}})| \psi_{0}(\overline{\boldsymbol{r}} ; \overline{\boldsymbol{R}})\right\rangle\right|^{2} \\
& \times \delta\left(\epsilon_{f}(\overline{\boldsymbol{R}})-\epsilon_{0}(\overline{\boldsymbol{R}})-\hbar \omega\right) .
\end{aligned}
$$

To calculate $\mathcal{O}(D \overline{\boldsymbol{r}})$ in Eq. A13, we use Eq. A3 for the XAS transition operator. In Eq. A3, gradient $\nabla$ is translation invariant and $\boldsymbol{t}$ applied on term $e^{i \boldsymbol{k} \cdot \boldsymbol{r}}$ only brings a phase $e^{i \boldsymbol{k} \cdot \boldsymbol{t}}$, which disappears in the modulus in Eq. A13.

Then, using the identity

$$
\boldsymbol{q} \cdot(\mathcal{S} \overline{\boldsymbol{r}})=\left(\mathcal{S}^{-1} \boldsymbol{q}\right) \cdot \overline{\boldsymbol{r}},
$$

with $\boldsymbol{q}=\hat{\boldsymbol{\varepsilon}}$ and $\boldsymbol{q}=\boldsymbol{k}$, we obtain the following symmetry property of the configuration cross section: ${ }^{70}$

$$
\sigma_{D \overline{\boldsymbol{R}}}(\hbar \omega, \hat{\varepsilon}, \boldsymbol{k})=\sigma_{\overline{\boldsymbol{R}}}\left(\hbar \omega, \mathcal{S}^{-1} \hat{\boldsymbol{\varepsilon}}, \mathcal{S}^{-1} \boldsymbol{k}\right) .
$$


Second, we examine the effect of applying $D$ onto $\overline{\boldsymbol{R}}$ in the $H_{0}\left(\overline{\boldsymbol{R}}, \overline{\boldsymbol{R}}_{0}\right)$ harmonic nuclear Hamiltonian, defined by Eq. A7 for $m=0$. The invariance of $\boldsymbol{r} \cdot \nabla$ under invertible linear transformations, the translation invariance of $\boldsymbol{R}_{I}-$ $\boldsymbol{R}_{I}^{0}$ and $\nabla$, and Eq. A12 imply

$$
H_{0}\left(D \overline{\boldsymbol{R}}, D \overline{\boldsymbol{R}}_{0}\right)=H_{0}\left(\overline{\boldsymbol{R}}, \overline{\boldsymbol{R}}_{0}\right)
$$

for any isometry $D$. If, moreover, $D$ is a symmetry element of the space group of the crystal, then $D \overline{\boldsymbol{R}}_{0}=\overline{\boldsymbol{R}}_{0}$ and

$$
H_{0}\left(D \overline{\boldsymbol{R}}, \overline{\boldsymbol{R}}_{0}\right)=H_{0}\left(\overline{\boldsymbol{R}}, \overline{\boldsymbol{R}}_{0}\right) .
$$

As a consequence, $\chi_{0}^{i}(\overline{\boldsymbol{R}})$ and $\chi_{0}^{i}(D \overline{\boldsymbol{R}})$ are eigenstates of the same Hamiltonian and, if they are non-degenerate, $\chi_{0}^{i}(D \overline{\boldsymbol{R}})$ and $\chi^{i}(\overline{\boldsymbol{R}})$ are equal up to a phase: ${ }^{71}$

$$
\left|\chi_{0}^{i}(D \overline{\boldsymbol{R}})\right|^{2}=\left|\chi_{0}^{i}(\overline{\boldsymbol{R}})\right|^{2} .
$$

Besides, $\chi_{0}^{i}(D \overline{\boldsymbol{R}})$ and $\chi_{0}^{i}(\overline{\boldsymbol{R}})$ have the same energy $E_{0}^{i}$ and thus, the same Boltzmann weight in Eq. A1.

Finally, we can investigate the average XAS cross section given by Eq. A8 when the space group symmetry operation $D$ is applied onto $\overline{\boldsymbol{R}}$. Using symmetry properties A17 and A15, we see that

$$
\begin{gathered}
\int d \overline{\boldsymbol{R}}\left|\chi_{0}^{i}(D \overline{\boldsymbol{R}})\right|^{2} \sigma_{D \overline{\boldsymbol{R}}}(\hbar \omega, \hat{\varepsilon}, \boldsymbol{k})= \\
\int d \overline{\boldsymbol{R}}\left|\chi_{0}^{i}(\overline{\boldsymbol{R}})\right|^{2} \sigma_{\overline{\boldsymbol{R}}}\left(\hbar \omega, \mathcal{S}^{-1} \hat{\boldsymbol{\varepsilon}}, \mathcal{S}^{-1} \boldsymbol{k}\right),
\end{gathered}
$$

where $\mathcal{S}$ is the symmetry operation of the point group of the crystal corresponding to $D$. Introducing Eq. A18 in Eq. A10, we obtain

$$
\sigma_{\mathrm{av}}(\hbar \omega, \hat{\varepsilon}, \boldsymbol{k})=\sigma_{\mathrm{av}}\left(\hbar \omega, \mathcal{S}^{-1} \hat{\varepsilon}, \mathcal{S}^{-1} \boldsymbol{k}\right),
$$

where $\mathcal{S}$ is any operation of the point group of the crystal. This shows that the average XAS cross section, which takes quantum thermal effects of the nuclei into account, has the same angular dependence (i.e. symmetry properties) as the cross section calculated with nuclei at their equilibrium positions.

The proof of Eq. A19 was given here in the harmonic approximation. It can be extended to include non-harmonic terms but it cannot be generalized to any temperature because crystal symmetry can be modified when phase transitions occur.

\section{APPENDIX B}

In the present work, nuclear thermal fluctuations are explicitly accounted for in the theoretical spectra by averaging cross sections calculated for out-of-equilibrium atomic configurations in which the crystal symmetry is

\begin{tabular}{lcc}
\hline \hline Index $i$ & $\hat{\varepsilon}$ & $\boldsymbol{k}$ \\
\hline 0 & {$[0 \overline{1} 0]$} & $(\sin \psi, 0,-\cos \psi)$ \\
1 & {$[1 \overline{1} 0]$} & $\left(\frac{-1}{\sqrt{2}} \sin \psi, \frac{1}{\sqrt{2}} \sin \psi,-\cos \psi\right)$ \\
2 & {$[100]$} & $(0, \sin \psi,-\cos \psi)$ \\
3 & {$[\overline{1} \overline{1} 0]$} & $\left(\frac{1}{\sqrt{2}} \sin \psi, \frac{-1}{\sqrt{2}} \sin \psi,-\cos \psi\right)$ \\
4 & {$[010]$} & $(-\sin \psi, 0,-\cos \psi)$ \\
5 & {$[\overline{1} 10]$} & $\left(\frac{-1}{\sqrt{2}} \sin \psi, \frac{-1}{\sqrt{2}} \sin \psi,-\cos \psi\right)$ \\
6 & {$[\overline{1} 00]$} & $(0,-\sin \psi,-\cos \psi)$ \\
7 & {$[110]$} & $\left(\frac{-1}{\sqrt{2}} \sin \psi, \frac{1}{\sqrt{2}} \sin \psi,-\cos \psi\right)$ \\
\hline \hline
\end{tabular}

TABLE II: Eight different $(\hat{\boldsymbol{\varepsilon}}, \boldsymbol{k})$ couples employed in the present calculations to extract the $\mathrm{D}_{4 \mathrm{~h}}$ in-plane mean and dichroic components of the $\mathrm{Ti} K$ edge in rutile $\mathrm{TiO}_{2}$ when accounting for thermal fluctuations. The angle $\psi$ specifies the direction of the wavevector $\boldsymbol{k}$ in the plane perpendicular to $\hat{\varepsilon}$.

lost. However, as proved in Appendix A and in the absence of phase transition, this average XAS cross section should display the same angular dependence as the cross section calculated with nuclei at the equilibrium positions, i.e. calculated for the full crystal symmetry.

In this Appendix, we present a practical method designed to retrieve the proper angular dependence of the absorption cross section from a set of calculations carried out with distinct $(\hat{\varepsilon}, \boldsymbol{k})$ couples within a generic outof-equilibrium configuration. In the remainder, we will adopt the efficient formalism developed by Ch. Brouder in Ref. 37 based on spherical tensor operators. Let us consider first the E1 contribution to the orientationindependent absorption cross section for a crystal with $\mathrm{D}_{4 \mathrm{~h}}$ point group and for a polarization normal to the fourfold rotation axis (i.e. $\hat{\varepsilon} \perp$ [001] direction). It reads (Eq. 4.4 of Ref. 37 with $\theta=\pi / 2$ )

$$
\sigma_{\mathrm{D}_{4 \mathrm{~h}}, \perp}^{\mathrm{E} 1}=\sigma^{\mathrm{D}}(0,0)+1 / \sqrt{2} \sigma^{\mathrm{D}}(2,0)
$$

whereas for a crystal with no symmetry, corresponding here to one of the configurations generated through a random displacement of the nuclear positions, it becomes (Eq. 4.7 of Ref. 37 with $\theta=\pi / 2$ )

$$
\begin{aligned}
\sigma_{\perp}^{\mathrm{E} 1}(\varphi)= & \sigma^{\mathrm{D}}(0,0)+1 / \sqrt{2} \sigma^{\mathrm{D}}(2,0) \\
& -\sqrt{3}\left[\cos 2 \varphi \sigma^{\mathrm{Dr}}(2,2)+\sin 2 \varphi \sigma^{\mathrm{Di}}(2,2)\right],
\end{aligned}
$$

where $\varphi$ is the azimuthal angle appearing in the spherical coordinates of $\hat{\varepsilon}$. The residual E1 dichroic signal arises therefore directly from the term in brackets in the right side of Eq. B2. A linear combination of two absorption cross sections only, calculated with suitable orientations of the polarization, can easily be built to cancel this term, restoring the in-plane isotropy of the electric dipole transitions

$$
\sigma_{\mathrm{D}_{4 \mathrm{~h}}, \perp}^{\mathrm{E} 1}=\frac{1}{2}\left[\sigma_{\perp}^{\mathrm{E} 1}\left(\hat{\varepsilon}_{i}\right)+\sigma_{\perp}^{\mathrm{E} 1}\left(\hat{\varepsilon}_{i+2}\right)\right]
$$


The different $\left(\hat{\varepsilon}_{i}, \boldsymbol{k}_{i}\right)$ couples employed in the present calculations are summarized in Table II. In the simple case of Eq. B3, all possibilities obtained, for instance, with $i=0,1, \ldots, 5$ are equivalent. Equations are slightly more cumbersome for E2 transitions but follow exactly the same lines. The absorption cross section for a crystal with $\mathrm{D}_{4 \mathrm{~h}}$ point group reads (Eq. 5.7 of Ref. 37 with $\theta=$ $\pi / 2)$

$$
\begin{aligned}
\sigma_{\mathrm{D}_{4 \mathrm{~h}}, \perp}^{\mathrm{E} 2}(\varphi, \psi)= & \sigma^{\mathrm{Q}}(0,0)+\sqrt{5 / 14}\left(3 \sin ^{2} \psi-1\right) \sigma^{\mathrm{Q}}(2,0) \\
& +1 / \sqrt{14}\left(5 \sin ^{2} \psi-4\right) \sigma^{\mathrm{Q}}(4,0) \\
& -\sqrt{5} \sin ^{2} \psi \cos 4 \varphi \sigma^{\mathrm{Qr}}(4,4),
\end{aligned}
$$

and leads to the non-zero E2 in-plane dichroic signal

$$
\sigma_{\mathrm{D}_{4 \mathrm{~h}}}^{\mathrm{dic}}=2 \sqrt{5} \sin ^{2} \psi \sigma^{\mathrm{Qr}}(4,4)
$$

observed experimentally on the pre-edge structures and obtained as the difference between Eq. B4 calculated with $\varphi$ equal to $\pi / 4$ and 0 . In Eq. B5, $\psi$ specifies the direction of the wavevector $\boldsymbol{k}$ in the plane perpendicular to $\hat{\varepsilon}$. For a crystal with no symmetry, the E2 contribution to the absorption cross section is given by (Eq. A.38 of Ref. 37 with $\theta=\pi / 2$ )

$$
\begin{aligned}
\sigma_{\perp}^{\mathrm{E} 2}(\varphi, \psi)= & \sigma^{\mathrm{Q}}(0,0)+\sqrt{5 / 14}\left(3 \sin ^{2} \psi-1\right) \sigma^{\mathrm{Q}}(2,0)+1 / \sqrt{14}\left(5 \sin ^{2} \psi-4\right) \sigma^{\mathrm{Q}}(4,0) \\
& +2 \sqrt{15 / 7} \sin \psi \cos \psi\left[\sin \varphi \sigma^{\mathrm{Qr}}(2,1)-\cos \varphi \sigma^{\mathrm{Qi}}(2,1)\right]-\sqrt{15 / 7} \cos ^{2} \psi\left[\cos 2 \varphi \sigma^{\mathrm{Qr}}(2,2)+\sin 2 \varphi \sigma^{\mathrm{Qi}}(2,2)\right] \\
& +\sqrt{10 / 7} \sin \psi \cos \psi\left[\sin \varphi \sigma^{\mathrm{Qr}}(4,1)-\cos \varphi \sigma^{\mathrm{Qi}}(4,1)\right]+2 \sqrt{5 / 7} \cos ^{2} \psi\left[\cos 2 \varphi \sigma^{\mathrm{Qr}}(4,2)+\sin 2 \varphi \sigma^{\mathrm{Qi}}(4,2)\right] \\
& -\sqrt{10} \sin \psi \cos \psi\left[\sin 3 \varphi \sigma^{\mathrm{Qr}}(4,3)-\cos 3 \varphi \sigma^{\mathrm{Qi}}(4,3)\right]-\sqrt{5} \sin ^{2} \psi\left[\cos 4 \varphi \sigma^{\mathrm{Qr}}(4,4)+\sin 4 \varphi \sigma^{\mathrm{Qi}}(4,4)\right] .
\end{aligned}
$$

Additional terms arising from the loss of symmetry between Eq. B4 and Eq. B6 can be cancelled through a linear combination of cross sections calculated for four distinct $(\hat{\boldsymbol{\varepsilon}}, \boldsymbol{k})$ couples

$$
\begin{aligned}
\sigma_{\perp, D_{4 \mathrm{~h}}}^{\mathrm{E} 2}\left(\hat{\boldsymbol{\varepsilon}}_{i}, \hat{\boldsymbol{k}}_{i}\right)= & \frac{1}{4}\left[\sigma_{\perp}^{\mathrm{E} 2}\left(\hat{\boldsymbol{\varepsilon}}_{i}, \hat{\boldsymbol{k}}_{i}\right)+\sigma_{\perp}^{\mathrm{E} 2}\left(\hat{\boldsymbol{\varepsilon}}_{i+2}, \hat{\boldsymbol{k}}_{i+2}\right)\right. \\
& \left.+\sigma_{\perp}^{\mathrm{E} 2}\left(\hat{\boldsymbol{\varepsilon}}_{i+4}, \hat{\boldsymbol{k}}_{i+4}\right)+\sigma_{\perp}^{\mathrm{E} 2}\left(\hat{\boldsymbol{\varepsilon}}_{i+6}, \hat{\boldsymbol{k}}_{i+6}\right)\right] .
\end{aligned}
$$

The E2 in-plane dichroic signal (Eq. B5) is then simply evaluated as the difference between Eq. B7 calculated for index $i=0$ and $i=1$, as given in Table II.

\footnotetext{
* Electronic address: sorbonne-universite.fr

guillaume.radtke@

1 O. Durmeyer, J.P. Kappler, E. Beaurepaire, J.M. Heintz and M. Drillon J. Phys.: Condens. Matter 2, 6127 (1990).

2 S.-J. Hwang, J.-H. Choy and N.H. Hur, Phys. Rev. B 57, 1259 (1998).

${ }^{3}$ N.L. Saini, A. Lanzara, H. Oyanagi, H. Yamaguchi, K. Oka, T. Ito and A. Bianconi, Phys. Rev. B 55, 12759 (1997).

4 Y.K. Kim, W.S. Jung, G.R. Han, K.-Y. Choi, C.-C. Chen, T.P. Devereaux, A. Chainani, J. Miyawaki, Y. Takata, Y. Tanaka, M. Oura, S. Shin, A.P. Singh, H.G. Lee, J.-Y. Kim, and C. Kim, Phys. Rev. Lett. 111, 217001 (2013).

${ }^{5}$ F. Bridges, C.H. Booth, G.H. Kwei, J.J. Neumeier and G. A. Sawatzky, Phys. Rev. B 61, R9237 (2000).

6 A.Y. Ignatov, N. Ali and S. Khalid, Phys. Rev. B 64, 014413 (2001).

7 P. Ghigna, A. Carollo, G. Flor, L. Malavasi, and G. Subias Peruga, J. Phys. Chem. B 109, 4365 (2005).

8 A. Yoshiasa, T. Nakatani, T. Hiratoko, T. Tobase, A.
}

Nakatsuka, M. Okube, H. Arima and K. Sugiyama, J. Phys.: Conference Series 712, 012121 (2016).

9 A. Yoshiasa, G. Kitahara, T. Tobase, T. Hiratoko, H. Hongu, T. Nakatani, and K.-I. Murai, Phys. Status Solidi $B$ 255, 1800050 (2018).

10 E. Cockayne, E.L. Shirley, B. Ravel, and J.C. Woicik, Phys. Rev. B 98, 014111 (2018).

11 I. Jonane, A. Cintins, A. Kalinko, R. Chernikov, A. Kuzmin, Low temp. phys. 44, 434 (2018).

12 I. Jonane, A. Anspoks, G. Aquilanti, A. Kuzmin, Acta Mat. 179, 26 (2019).

13 U. Bergmann, P. Glatzel and S.P. Cramer, Microchemical Journal 71, 221 (2002).

${ }^{14}$ C. Sternemann and M. Wilke, High Press. Res. 36, 275 (2016).

15 S. K. Lee, P. J. Eng, H.-K. Mao, Y. Meng, M. Newville, M. Y. Hu, and J. Shu, Nat. Mater. 4, 851 (2005).

16 J.-F. Lin, H. Fukui, D. Prendergast, T. Okuchi, Y. Q. Cai, N. Hiraoka, C.-S. Yoo, A. Trave, P. Eng, M. Y. Hu, and P. Chow, Phys. Rev. B 75, 012201 (2007). 
17 G. Lelong, L. Cormier, G. Ferlat, V. Giordano, G. S. Henderson, A. Shukla, and G. Calas, Phys. Rev. B 85, 134202 (2012).

18 J.S. Tse, M. Hanfland, R. Flacau, S. Desgreniers, Z. Li, K. Mende, K. Gilmore, A. Nyrow, M. Moretti Sala, and C. Sternemann, J. Phys. Chem. C 118, 1161 (2014).

19 H. Conrad, F. Lehmkühler, C. Sternemann, A. Sakko, D. Paschek, L. Simonelli, S. Huotari, O. Feroughi, M. Tolan and K. Hämäläinen, Phys. Rev. Lett. 103, 218301 (2009).

20 C.J. Sahle, C. Sternemann, C. Schmidt, S. Lehtola, S. Jahn, L. Simonelli, S. Huotari, M. Hakala, T. Pylkkänen, A. Nyrow, K. Mende, M. Tolan, K. Hämäläinen, and M. Wilke, Proc. Natl. Acad. Sci. (U.S.A.) 110, 6301 (2013).

${ }^{21}$ C.J. Sahle, S. Kujawski, A. Remhof, Y. Yan, N.P. Stadie, A. Al-Zein, M. Tolan, S. Huotari, M. Krisch and C. Sternemann, Phys. Chem. Chem. Phys. 18, 5397 (2016).

22 D. Cabaret and C. Brouder, J. Phys.: Conf. Ser. 190, 012003 (2009).

23 C. Brouder, D. Cabaret, A. Juhin, and P. Sainctavit, Phys. Rev. B 81, 115125 (2010).

24 D. Manuel, D. Cabaret, Ch. Brouder, Ph. Sainctavit, A. Bordage, and N. Trcera, Phys. Rev. B 85, 224108 (2012).

25 A.L. Ankudinov and J.J. Rehr, Phys. Scrip. T115, 24 (2005).

26 T. Fujikawa, J. Phys. Soc. Jpn. 68, 2444 (1999).

27 T.A. Pascal, U. Boesenberg, R. Kostecki, T.J. Richardson, T.-C. Weng, D. Sokaras, D. Nordlund, E. McDermott, A. Moewes, J. Cabana and D. Prendergast, J. Chem. Phys. 140, 034107 (2014)

28 P. Müller, K. Karhan, M. Krack, U. Gerstmann, W. Gero Schmidt, M. Bauer, and T.D. Kühne, J. Comput. Chem. 40, 712 (2019).

29 M.P. Ljungberg, J.J. Mortensen and L.G.M. Pettersson, J. Electron Spectrosc. Relat. Phenom. 184, 427 (2011).

30 R. Nemausat, D. Cabaret, C. Gervais, C. Brouder, N. Trcera, A. Bordage, I. Errea and F. Mauri, Phys. Rev. B 92, 144310 (2015).

${ }^{31}$ R. Nemausat, C. Gervais, C. Brouder, N. Trcera, A. Bordage, C. Coelho-Diogo, P. Florian, A. Rakhmatullin, I. Errea, L. Paulatto, M. Lazzeri and D. Cabaret, Phys. Chem. Chem. Phys. 19, 6246 (2017).

32 D. Cabaret, A. Bordage, A. Juhin, M. Arfaoui and E. Gaudry, Phys. Chem. Chem. Phys. 12, 5619 (2010).

33 F.M.F. de Groot, X-ray Absorption Fine StructureXAFS13, AIP Conference Proceedings, 882, 37 (2007).

${ }^{34}$ W. Schülke, Electron Dynamics by Inelastic X-ray Scattering, Oxford Series on Synchrotron Radiation No. 7 (Oxford University Press, Oxford, 2007)

35 E. de Clermont Gallerande, D. Cabaret, G. Lelong, C. Brouder, M.-B. Attaiaa, L. Paulatto, K. Gilmore, Ch.J. Sahle and G. Radtke, Phys. Rev. B 98, 214104 (2018).

36 Y. Joly, C. Cavallari, S.A. Guda and C.J. Sahle, J. Chem. Theory and Comput. 13, 2172 (2017).

37 C. Brouder, J. Phys.: Condens. Matter 2, 701 (1990).

38 S. Baroni, S. de Gironcoli, A. Dal Corso and P. Giannozzi, Rev. Mod. Phys. 73, 515 (2001).

39 Y. Joly, D. Cabaret, H. Renevier and C.R. Natoli Phys. Rev. Lett. 82, 2398 (1999).

${ }^{40}$ G. Beutier, S.P. Collins, G. Nisbet, E.N. Ovchinnikova and V.E. Dmitrienko, Eur. Phys. J. Special Topics 208, 53 (2012).

41 S. Huotari, C. J. Sahle, C. Henriquet, A. Al-Zein, K. Martel, L. Simonelli, R. Verbeni, H. Gonzalez, M.-C. Lagier, C. Ponchut, M. Moretti Sala, M. Krisch and G. Monaco,
J. Synchrotron Rad. 24, 521 (2017).

42 P. Giannozzi, S. Baroni, N. Bonini, M. Calandra, R. Car, C. Cavazzoni, D. Ceresoli, G. L. Chiarotti, M. Cococcioni, I. Dabo, A. Dal Corso, S. de Gironcoli, S. Fabris, G. Fratesi, R. Gebauer, U. Gerstmann, Ch. Gougoussis, A. Kokalj, M. Lazzeri, L. Martin-Samos, N. Marzari, F. Mauri, R. Mazzarello, S. Paolini, A. Pasquarello, L. Paulatto, C. Sbraccia, S. Scandolo, G. Sclauzero, A.P. Seitsonen, A. Smogunov, P. Umari and R.M. Wentzcovitch, J. Phy.: Condens. Mat. 21, 395502 (2009).

43 P. Giannozzi, O. Andreussi, T. Brumme, O. Bunau, M. Buongiorno Nardelli, M. Calandra, R. Car, C. Cavazzoni, D. Ceresoli, M. Cococcioni, N. Colonna, I. Carnimeo, A. Dal Corso, S. de Gironcoli, P. Delugas, R.A. DiStasio, A. Ferretti, A. Floris, G. Fratesi, G. Fugallo, R. Gebauer, U. Gerstmann, F. Giustino, T. Gorni, J. Jia, M. Kawamura, H.-Y. Ko, A. Kokalj, E. Kçkbenli, M. Lazzeri, M. Marsili, N. Marzari, F. Mauri, N.L. Nguyen, H.-V. Nguyen, A. Otero-de-la-Roza, L. Paulatto, S. Poncé, D. Rocca, R. Sabatini, B. Santra, M. Schlipf, A.P. Seitsonen, A. Smogunov, I. Timrov, T. Thonhauser, P. Umari, N. Vast, X. Wu and S. Baroni, J. Phy.: Condens. Mat. 29, 465901 (2017).

44 M. Taillefumier, D. Cabaret, A.M. Flank and F. Mauri, Phys. Rev. B 66, 195107 (2002).

${ }^{45}$ Ch. Gougoussis, M. Calandra, A.P. Seitsonen and F. Mauri, Phys. Rev. B 80, 075102 (2009).

46 D. Vanderbilt, Phys. Rev. B 41, 7892 (1990).

47 A. Dal Corso, Comput. Mater. Sci. 95, 337 (2014).

48 A. M. Rappe, K. M. Rabe, E. Kaxiras, and J. D. Joannopoulos, Phys. Rev. B 41, 1227 (1990).

49 J.P. Perdew, K. Burke and M. Ernzerhof, Phys. Rev. Lett. 77, 3865 (1996).

50 B. Montanari and N.M. Harrison, Chem. Phys. Lett., 364, 528 (2002).

${ }^{51}$ K. Refson, B. Montanari, P.D. Mitev, K. Hermansson and N.M. Harrison, Phys. Rev. B 88, 136101 (2013).

52 J.P. Perdew and A. Zunger, Phys. Rev. B 23, 5048 (1981).

${ }^{53}$ G. Fiquet, P. Richet and G. Montagnac Phys. Chem. Minerals 27, 103 (1999).

${ }^{54}$ G. Lelong, G. Radtke, L. Cormier, H. Bricha, J.P. Rueff, J.M. Ablett, D. Cabaret, F. Gélébart and A. Shukla, Inorg. Chem. 53, 10903 (2014).

55 S. Baroni, P. Giannozzi and E. Isaev, Rev. Mineral. Geochem. 71, 39 (2010).

56 D.W. Fischer, Phys. Rev. B 5, 4219 (1972).

57 B. Poumellec, R. Cortes, G. Tourillon and J. Berthon, Phys. Status Solidi B 164, 319 (1991).

58 T. Uozumi, K. Okada, A. Kotani, O. Durmeyer, J.P. Kappler, E. Beaurepaire and J.C. Parlebas, Europhys. Lett. 18, 85 (1992).

59 E. Beaurepaire, S. Lewonczuk, J. Ringeissen, J.C. Parlebas, T. Uozumi, K. Okada and A. Kotani, Europhys. Lett. 22, 463 (1993).

60 D. Cabaret, Y. Joly, H. Renevier and C.R. Natoli J. Synchrotron Rad. 6, 258 (1999).

61 E.L. Shirley, J. Electron Spectrosc. Relat. Phenom. 136, 77 (2004)

${ }^{62}$ P. Le Fèvre, H. Magnan, D. Chandesris, J. Jupille, S. Bourgeois, A. Barbier, W. Drube, T. Uozumi, and A. Kotani, Nucl. Instrum. Methods Phys. Res. A 547, 176 (2005).

63 Z.Y. Wu, G. Ouvrard, P. Gressier, and C.R. Natoli, Phys. Rev. B 55, 10382 (1997).

${ }^{64}$ We used a local orthonormal system of coordinates ori- 
ented along the two-fold rotation axes of the $\mathrm{D}_{2 \mathrm{~h}}$ point group of the Ti site. We arbitrarily chose $\hat{x}$ to point along [001] and $\hat{z}$ along [110] and considered the Ti located at $(0,0,0)$. With this choice, the $\hat{z}$ axis is oriented along the $1.98 \AA \mathrm{Ti}-\mathrm{O}$ bond and $\hat{x}$ and $\hat{y}$ point toward the middle of the edges of the rectangle formed by the four equatorial O ions distant from the central Ti by $1.94 \AA$. Accordingly, real $d$ orbitals $d_{x^{2}-y^{2}}, d_{x z}, d_{y z}, d_{z^{2}}$ and $d_{x y}$ transform as the irreducible representations $A_{g}, B_{2 g}, B_{3 g}, A_{g}$ and $B_{1 g}$, respectively.

${ }^{65}$ R. Brydson, H. Sauer, W. Engel, J.M. Thomass, E. Zeitler, N. Kosugi and H. Kuroda, J. Phys. : Condens. Matter 1, 797 (1989).

66 T. C. Rossi, D. Grolimund, O. Cannelli, G.F. Mancini, C. Bacellar, D. Kinschel, J.R. Rouxel, N. Ohannessian, D. Pergolesic and M. Chergui J. Synchrotron Rad. 27, 425
(2020).

67 S. Nozawa, T. Iwazumi and H. Osawa, Phys. Rev. B 72, 121101(R) (2005).

68 M. Chassé, A. Juhin, D. Cabaret, S. Delhommaye, D. Vantelon and G. Calas Phys. Chem. Chem. Phys. 20, 23903 (2018).

${ }^{69}$ If the eigenvalues are degenerate, the set of degenerate states is a basis of a unitary representation of the group and the final result is the same.

70 Similarly in XRS, the transition operator being $\mathcal{O}(\boldsymbol{r})=$ $e^{i \boldsymbol{q} \cdot \boldsymbol{r}}$, translations do not affect the dynamic structure factor and we have again $S_{D \overline{\boldsymbol{R}}}(\hbar \omega, \boldsymbol{q})=S_{\overline{\boldsymbol{R}}}\left(\hbar \omega, \mathcal{S}^{-1} \boldsymbol{q}\right)$.

${ }^{71}$ If $\chi_{0}^{i}(D \overline{\boldsymbol{R}})$ and $\chi^{i}(\overline{\boldsymbol{R}})$ are degenerate, the set of degenerate states is a basis of a unitary representation of the group and the final result is the same. 